\author{
Universidade de São Paulo \\ Instituto de Física
}

\title{
MHD EQUILIBRIUM IN TOKAMAKS With ReVersed CurRent Density
}

\author{
David Ciro Taborda
}

Orientador: Prof. Dr. Iberê Luiz Caldas

Dissertação de mestrado apresentada ao Instituto de Física para a obtenção do título de Mestre em Ciências.

Prof. Dr. Iberê Luiz Caldas - IFUSP (Orientador)

Prof. Dr. Ricardo Magnus Osorio Galvão - IFUSP

Prof. Dr. Marisa Roberto - ITA 
FICHA CATALOGRÁFICA

Preparada pelo Serviço de Biblioteca e Informação do Instituto de Física da Universidade de São Paulo

Taborda, David Ciro

Equilibrio magnetohidrodinâmico em plasmas com densidade de corrente reversa. São Paulo, 2012.

Dissertação (Mestrado) - Universidade de São Paulo. Instituto de Física, Depto. Física Aplicada

Orientador: Prof. Dr. Iberê Luiz Caldas

Área de Concentração: Equilibrio Magnetohidrodinâmico

Unitermos: 1. Magnetohidrodinâmica; 2. Sistemas

dinâmicos; 3. Teoria eletromagnética; 4. Tokamaks. 
Este trabalho não teria sido possível sem a ajuda, orientação e apoio das pessoas que me acompanham dia-a-dia em pensamento ou corpo presente.

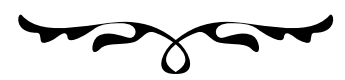

Ao Prof. Dr. Iberê Luiz Caldas, por me receber no seu grupo de pesquisa, compartilhar comigo sua experiencia e me apresentar a Física de Plasmas. Pela excelente orientação e crítica.

Aos meus companheiros: Julio, Celso, Dennis, René, Thiago, Rafael, Everton e Meirielen pela amizade, a constante troca de ideias e a cálida recepção no grupo de trabalho.

\section{sto}

Ao Prof. Dr. Ricardo M. Galvão, pelas rigorosas discussões e recomendações. Pelo seu tempo e disposição.

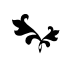

A Sheron, por me acompanhar neste caminho e estar sempre presente. Pela felicidade que me traz.

\section{$7 x^{2}$}

A mis padres, Estella y Edilberto, y al resto de la Familia, por estar siempre en mi corazón, por darme la fuerza y el orgullo con que abro mi camino.

\section{$7 x^{2}$}

A los viejos amigos, que no caben en estas páginas y llenan mis recuerdos.

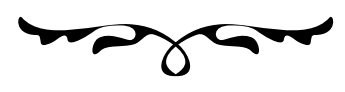

Obrigado - Gracias. 


\section{AKNOWLEDMENTS}

It is worth recognizing the national concern in the development of basic and applied sciences, as well as the open inclusion of foreign neighbors. Such attitude constitutes an important motive force in the region's development.

Thanks to the Physics Institute of Universidade de São Paulo, for providing the structure and conditions that made possible this work. This research has been developed under financial support of CNPq, process 130250/2011-2. 


\section{Abstract}

In the present work, Current Reversal Equilibrium Configurations (CRECs) in the context of Magnetohydrodinamic (MHD) equilibrium are considered. The hamiltonian nature of the magnetic field lines is used to introduce the concept of magnetic surfaces and their

relation to the Grad-Shafranov (G-S) equation. From a geometrical perspective and the Maxwell equations, it is shown that current reversal configurations in two-dimensional equilibrium do not generate the usual nested topology of the equilibrium magnetic surfaces. The concept of intersecting critical curves is introduced to describe the CRECs and recently published equilibria are shown to be compatible with such description. The equilibrium with a single magnetic island is constructed analytically, through a local successive approximations method, valid for any choice of the source functions of the G-S equation. From the local solution, an estimate of the island width in terms of simple quantities is deduced and verified to a good accuracy with recently published CRECs; the accuracy of this simple model suggests the existence of strong topological constraints in the formation of the equilibria. Lastly, an instability mechanism is conjectured to explain the lack of conclusive experimental evidence of reversed currents, in favor of the current clamp hypothesis. 


\section{RESUMO}

No presente trabalho, as configurações de equilíbrio com corrente reversa (CRECs), são consideradas no contexto de Equilíbrio Magnetoidrodinâmico. A natureza hamiltoniana das linhas de campo magnético é usada para introduzir o conceito de superfícies magnéticas, e sua relação com a equação de Grad-Shafranov (G-S). Desde uma perspectiva geométrica e usando as equações de Maxwell, é demonstrado que as configurações de corrente reversa em equilíbrios bidimensionais não é compativel com as topologias aninhadas usuais para as superfícies magnéticas de equilíbrio. O conceito de curvas críticas é introduzido para descrever as CRECs e é observado que os equilíbrios recetemente publicados satisfazem esta descrição. O equilíbrio com uma única ilha magnética é construído analiticamente, por meio de aproximações sucessivas locais, este é válido para qualquer escolha das funções arbitrárias da equação G-S. A partir da solução local, se desenvolve uma estimativa do tamanho da ilha magnética em termos de quantidades simples. Esta estimativa concorda bem com as CRECs da literatura recente, sugerindo pela simplicidade do modelo, que existem fortes restrições topológicas no estabelecimento do equilíbrio. Finalmente, na forma de conjectura, introduzimos um mecanismo para instabilidades que tenta dar conta da falta de evidência experimental conclusiva em relação às CRECs em favor da hipótese de corrente unidirecional (current clamp). 


\section{Contents}

1 Introduction 13

2 Tokamaks and Magnetic Fields 17

2.1 Toroidal Geometry . . . . . . . . . . . . . . . . . . . 17

2.2 Need of External Fields . . . . . . . . . . . . . . . . . . . . . . . . . . . . . 19

2.3 Nature of Magnetic Fields . . . . . . . . . . . . . . . . . . . 20

2.3.1 Passive tracers . . . . . . . . . . . . . . . . . 20

2.3.2 Divergenceless Fields . . . . . . . . . . . . . . . . . . . . . . . 21

2.3.3 Hamiltonian Magnetic Lines . . . . . . . . . . . . . . . . . . . . . 22

2.4 Hamiltonian Systems . . . . . . . . . . . . . . . . . . 23

3 MHD EqUILIBRIUM 27

3.1 Single Fluid Equations . . . . . . . . . . . . . . . . . . . . . . . 28

3.2 Equilibrium . . . . . . . . . . . . . . . . . . . . . . . 29

3.2.1 Grad-Shafranov Equation . . . . . . . . . . . . . . 31

3.3 Equilibrium Solutions . . . . . . . . . . . . . . . . . . . . . 36

3.3.1 Boundary Conditions . . . . . . . . . . . . . . . 36

3.3.2 Successive Approximations . . . . . . . . . . . . . . . . . . 38

3.3.3 Safety Factor . . . . . . . . . . . . . . . . . . . . . . 41

4 Current Reversal Equilibrium Results 43

4.1 Comments on Stability . . . . . . . . . . . . . . . . . 44

4.2 Topology of the CRECs . . . . . . . . . . . . . . . 45

4.2 .1 Generalization . . . . . . . . . . . . . . . . . . 48

$4.2 .2 \quad$ Non-simple circuits . . . . . . . . . . . . . . . . . . . . . . . . . 49

4.3 Local Solution to the Grad-Shafranov Equation . . . . . . . . . . . . . 51

4.3 .1 Magnetic island width . . . . . . . . . . . . . . . 57

4.3 .2 Safety Factor . . . . . . . . . . . . . . . . . . . . 59

4.3.3 The current clamp hypothesis and the instability conjecture . . . . 62

4.4 Concluding Remarks . . . . . . . . . . . . . . . . 66 
5 Conclusions $\quad 67$

6 APPENDix $\quad 69$

6.1 Poloidal flux perturbation model . . . . . . . . . . . . . . . . 69

$\begin{array}{ll}\text { References } & 70\end{array}$ 


\section{Chapter 1}

\section{INTRODUCTION}

Fusion devices has been pursued by physicists since the early 1950's, when the concept of tokamak $^{1}$ was first introduced by A. Shakarov and I. Tamm [1]. In essence, a tokamak is a toroidal metallic chamber, immersed in a suitable array of magnetic fields. Those fields are intended to confine the charged particles of a ionized gas at high temperatures inside the chamber.

A successful fusion reactor must confine a sufficiently dense plasma at high temperatures for a considerable long time, so that we can extract thermonuclear power from it [2]. Such power is due to the energy difference, between the rest-energy of two light nuclei and the rest-energy of the resulting nucleus (lighter that its components) after fusion. The Deuterium-Tritium (D-T) reaction is considered most efficient for fusion devices. Deuterium occurs naturally, is widely abundant and can be efficiently extracted from water; meanwhile, Tritium can be produced from Lithium when submitted to a neutron beam, which is one sub-product of the fusion reaction. The recent Lithium Tokamak Experiment $^{2}$ (LTX) incorporates in its design a liquid Lithium plasma-facing component (PFC), that reduces impurities and the need of recycling, it also suggests an on-the-way Tritium production [3].

If sustained, fusion promises to be a clean, cheap and efficient source of energy and tokamaks appears have the most promising design for confinement. During the last 50 years, the tokamak has been improved, but the confinement problem has proven extremely difficult, involving several research fields. The self-sustained fusion reaction and stable confinement problem has then become an strictly interdisciplinary task, only solvable if many considerations take place.

The plasma state is a highly dynamical one, since atoms are split in their charged components that moves in response to the long range Coulomb forces produced by other

\footnotetext{
${ }^{1}$ Transliteration from the Russian, acronym for "Toroidal Chamber with Magnetic Coils".

${ }^{2}$ See http://pst.pppl.gov/ltx/
} 
charged particles in the system. Now, the self-consistency problem comes due to the electromagnetic field produced by the moving charges, that affects back their own orbits, creating thus a very complex picture of a magnetohydrodynamic system. In general we have a nonlinear free-boundary problem, since the shape of the plasma border depends strongly on its internal configuration, therefore it must be found self-consistently. Also, the study of plasma dynamics involves several time scales and strong assumptions about the regime of the system are mandatory.

Although the plasma state generally changes in time, one of the most fundamental questions is about its equilibrium configurations. This comes as a generalization of the basic method in Dynamical Systems Theory, where the fixed points on the phase space give important information about the topology of the phase flow, in addition to its stability in the nearby regions. Then, before undertake any serious attempt to solving the general magnetohydrodynamic problem, the equilibrium configurations must be known and their stability parameters as well. In this context, the Grad-Shafranov (G-S) equation appears as a cornerstone of the equilibrium theory for two-dimensional plasmas ${ }^{3}$; its solution provides the topology of the so called magnetic surfaces, where the equilibrium magnetic field winds on surfaces with constant poloidal magnetic flux. However to solve the G$\mathrm{S}$ equation, a couple of arbitrary functions must be provided and the relevant physical quantities depend strongly on this choice, establishing again self-consistency issues.

In general, after solving the G-S equation, we are able to describe the equilibrium through relevant scalar fields, like the pressure and current density. Knowing these, we can determine if the given equilibrium presents good features for a fusion reactor, as high energy confinement (pressure), and stability. In standard equilibria, monotonic current profiles decrease from the magnetic axis to the plasma edge, where the density of charged particles is expected to vanish. This configuration presents some desirable features for fusion, as a maximum pressure at the center, but has a strong dependence on the externally produced magnetic fields and its improvement is limited by stability considerations. Another fundamental issue comes from the basic mechanism to drive the toroidal current inside the tokamak, that relies on the electromotive force induced by a varying magnetic flux from an external transformer, limiting the tokamak operation, at constant plasma current, to the time required to ramp the external current to its saturation value. This, among other reasons, compels to search for advanced tokamak scenarios that may allow a continuous operation of fusion power plants. As expected, in those advanced scenarios, most of the plasma current is non-inductive [4], and other sources of current must be provided. For instance, the bootstrap current [5] is internally produced by particle trapping effects, due to variations in the magnetic field intensity

\footnotetext{
${ }^{3}$ Two-dimensional here refers to the structure of the problem when a symmetry is imposed.
} 
along the field lines. It is driven by the geometry of the equilibrium and the pressure gradients, which makes it naturally hollow, with an off-axis minimum. There are also externally driven currents, which can be pumped continuously, like the LH-driven current produced by externally excited Lower-Hybrid waves.

From the hollow nature of the bootstrap current, it is natural to think of advanced operation schemes involving non-monotonic current profiles. These are closely related to non-monotonic safety factors that have a number of advantages in regard to transport properties. In the context of advanced operation regimes, hollow current profiles have been studied experimentally [6-8], and high confinement due to internal transport barriers (ITB) was evidenced. In the context of axisymmetric equilibrium configurations, strong invariant surfaces appears when the safety factor has a non-monotonic profile [9]. This strong torus are robust against non-axisymmetric magnetic perturbations, reducing the magnetic lines transport that drives the charge carriers. The same effects are observed in related discrete models or nontwist mappings [10].

In recent works, the so called Current Reversal Equilibrium Configurations (CRECs), a natural extension of the hollow scenarios, allows a small part of the current density to become negative. The conjectured current reversal $[11,12]$ has been studied numerically and analytically. In both cases, non-nested structures appear around some critic magnetic surface, producing an equilibrium topology with a finite number of saddle points and magnetic islands. If a negative current density is attainable, then it is most probable that topological changes in the equilibrium may have a major influence in the viability of these configurations. Understanding of the conjectured current reversal is relevant to define the limits of advanced scenarios and their stability under current drive mechanisms.

An important issue of the known analytical descriptions of CRECs is the choice of a unique set of arbitrary source functions for solving the G-S equation in the whole domain. This approach is in general valid for nested topologies, but, in nonested equilibria, different families of nested surfaces exist, so that actually different source functions may apply to different families of nested magnetic surfaces. This introduces the extra difficulty, in a more rigorous equilibrium calculation, of establishing internal boundary conditions for self-consistency.

In the present work it is shown that any reversed current density is a mechanism for magnetic island formation in the axisymmetric case and that the relevant equilibrium geometry is almost independent of the choice of arbitrary sources. This can be understood directly from the fundamentally two-dimensional nature of the equilibrium. Then, the Grad-Shafranov equation is solved locally for a generic current profile containing the main desired features. This simple model reproduces the most relevant topology of the one-island cases reported in the literature. After recognizing the basic features of CRECs, 
their stability is discussed in the non-axisymmetric case, where some concepts from chaotic hamiltonian systems are used to lighten an apparently basic mechanism for instability.

The present work is organized as follows. In Chapter 2, the basic properties of magnetic fields and tokamaks are introduced; then in Chapter 3 a short digression in Magnetohydrodynamics and equilibrium theory introduces the basic tools to confront the problem of CRECs in Chapter 4, where the topology of equilibrium and its stability are studied. Chapter 5, contains some conclusions and a short discussion. 


\section{Chapter 2}

\section{Tokamaks And Magnetic FieldS}

\section{$2.1 \quad$ Toroidal Geometry}

Due to the high temperatures needed to maintain an ionized plasma, about $10^{9} \mathrm{~K}$ in a usual configuration, the confinement of plasmas by material walls is out of question, not because of heating them to melting temperatures, because plasma densities are usually low, between $10^{19}-10^{20} m^{-3}$ [13], but for the sputtering process that may take place, introducing heavy impurities in the plasma, and, eventually cooling it down below ignition. The idea of magnetic confinement comes from the fact that the orbits of charged particles curl in presence of magnetic fields, and to a first order approximation, their gyrocenter follows the magnetic field lines. This suggests that magnetic fields may be used to confine charged particles in a small volume (Fig 2.1), so that they can collide without going very far, therefore sustaining the density-temperature conditions for ignition to take place. The Lawson criterion defines the conditions needed to maintain the plasma temperature with the fusion products alone, balancing the energy losses without external power [14].

$$
n T \tau_{E}>3 \times 10^{21} \mathrm{KeVs} / \mathrm{m}^{3}
$$

where $n, T$ are the density and temperature, and $\tau_{E}$ is the confinement time given by

$$
\tau_{E}=\frac{\frac{3}{2} \int n\left(T_{i}+T_{e}\right)}{P},
$$

with $P$ the power input of the system and $i, e$ stands for ions and electrons ${ }^{1}$. This criterion, however, does not guarantee the sustainment of such configuration, only establishes a break-point for power production.

In order to simplify the confinement problem, the magnetic field configuration must

\footnotetext{
${ }^{1}$ Ions and electrons have different temperatures since collisions between them is less frequent that the electron-electron and ion-ion collisions.
} 


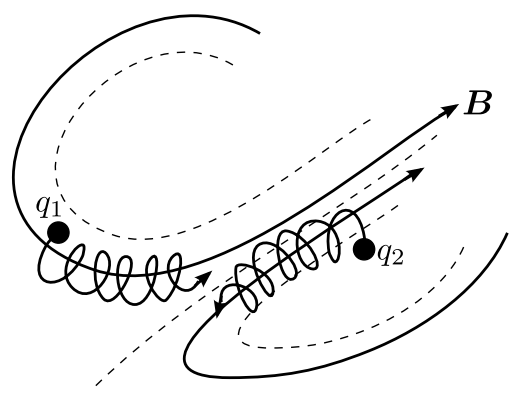

Figure 2.1: Magnetic lines driving charges to a collision.

be simple, to enhance the experimental control and reduce the number of parameters for the experimental setup. To understand the basic topology of tokamaks we just have to recall Ampere's law.

$$
\nabla \times \boldsymbol{B}=\mu_{0} \boldsymbol{j} \Rightarrow \oint_{\Gamma} \boldsymbol{B} \cdot d \boldsymbol{l}=\mu_{0} I
$$

In our context, we may think that the current density $\boldsymbol{j}$ is produced by the electronion flow inside the plasma, so that the net flux through a given open surface gives a net circulation of the magnetic field in its border. The most simple configuration is that of a straight cylinder of moving charges, producing a solenoidal field about it, but this defines an unbounded volume for the plasma. Thus we may simply blend the cylinder identifying both ends to define a closed volume containing the particles. This is the most simple geometry allowing a net circulation of the magnetic field about the plasma current. Now, from

$$
\nabla \cdot \boldsymbol{B}=0
$$

we know that there are no sources of magnetic field, so that the magnetic lines must always encircle the toroidal shape (Fig. 2.2).
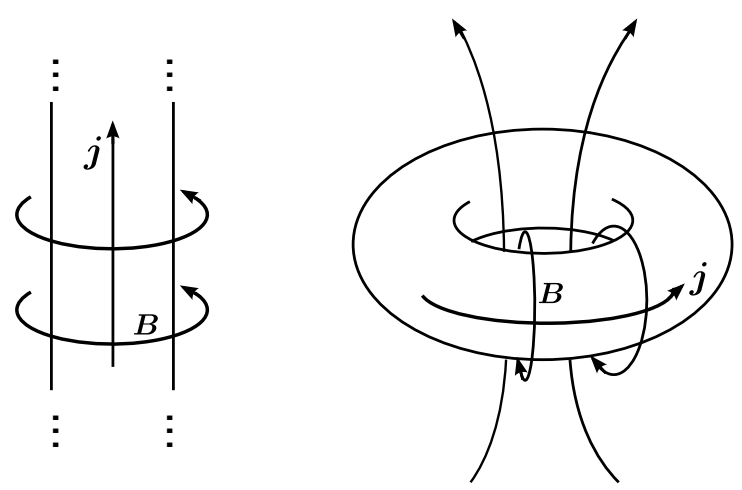

Figure 2.2: A toroidal current defines the most simple volume with net circulation of magnetic field around it. 


\section{$2.2 \quad$ Need of External Fields}

Until now we have thought of the plasma currents as sources of the magnetic field, however, it is easy to show that to confine the plasma we need external magnetic fields $[15,16]$. The most resumed equilibrium condition is given by

$$
\nabla \cdot \mathbb{T}=0
$$

with the definition,

$$
\mathbb{T}=p_{\perp}(\mathbb{I}-\boldsymbol{b} \boldsymbol{b})+p_{\|} \boldsymbol{b} \boldsymbol{b},
$$

where $\boldsymbol{b}=\frac{\boldsymbol{B}}{B}, p_{\perp}=p+\frac{1}{2} B^{2}, p_{\|}=p-\frac{1}{2} B^{2}$ and $p$ the plasma pressure. We can integrate over a given volume the expression $\nabla \cdot(\mathbb{T} \cdot \boldsymbol{r})=\operatorname{Tr}(\mathbb{T})+(\nabla \cdot \mathbb{T}) \cdot \boldsymbol{r}$, and after Gauss theorem is applied we get

$$
\int\left(3 p+B^{2} / 2\right) d V=\oint_{\sigma}\left[\left(p+B^{2} / 2\right) \boldsymbol{r} \cdot d \boldsymbol{S}-(\boldsymbol{B} \cdot \boldsymbol{r})(\boldsymbol{B} \cdot d \boldsymbol{S})\right] .
$$

In the left side, the integrand is positive definite and so is the integral. Now, if we take the containing surface to go to infinity, recalling that $B$ falls like $1 / r^{3}$ or faster, the surface integral vanishes and we get an absurd result. This comes from the equilibrium equation (2.5), that only considers fields produced by the plasma current, neglecting any external source of field. The conclusion is clear, the local equilibrium condition in absence of external fields is not compatible with the global equilibrium equations, then the equilibrium requires the introduction of external fields.

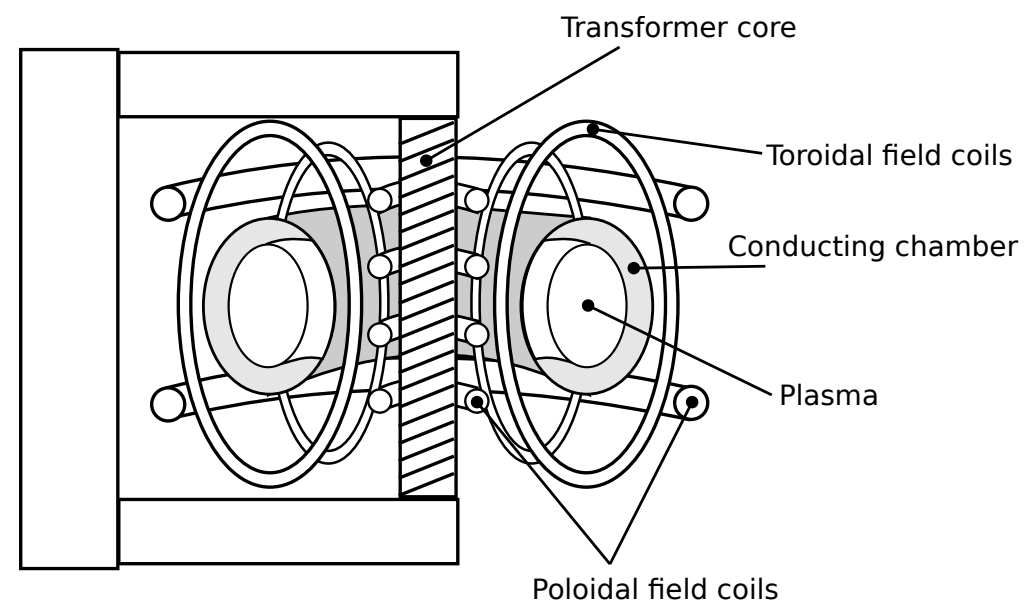

Figure 2.3: Basic design of a tokamak.

In Figure 2.3 we can see the basic coil structure, used to create the vacuum magnetic field where the plasma is introduced. A central iron core is part of a transformer used to create the toroidal plasma current by Faraday Induction. Also, the vacuum magnetic 
field has both poloidal and toroidal components, the latter are produced by a suitable set of current rings. In equilibrium, a consistent configuration of magnetic fields and plasma currents gives the desired confinement of charged particles.

\subsection{Nature of Magnetic Fields}

Until now, a combination of externally and internally produced magnetic fields appear to be the most fundamental tool to confine plasmas. Magnetic fields possesses remarkable geometric properties coming from its divergenceless nature, like an intrinsic symplectic structure. In the following, a short survey of fundamental properties relevant to this work will be presented.

\subsubsection{Passive tracers}

In Electrodynamics we study the structure of electromagnetic fields and their relation to the sources, charges and currents. The electromagnetic field is as an entity with physical properties of its own, but in practice, this fields serve to define the long-range forces acting on charged particles under the influence of other charges and currents, i.e. fields may be considered a means, not an end. Nonetheless, it is possible to show that, under some averaging conditions, the motion of charged particles in electromagnetic fields may be decomposed into a fast gyromotion about a slow guiding centre motion [17]. The guiding centre motion has its main component along the magnetic field ${ }^{2}$ and there are several drifts $(E \times B$, perpendicular, $\nabla B$, inertial and polarization $)$, driving the center off the magnetic lines.

By studying the magnetic field lines, we can understand to first order, the average motion of charged particles in a slowly varying (in space and time) electromagnetic field. For this, let us introduce the concept of passive tracers [18]. Assume a vector field $\boldsymbol{V}(\boldsymbol{r})$, and a set of three independent, arbitrary functions $\left\{u^{1}, u^{2}, u^{3}\right\}$; the vector field may be written in the covariant basis as ${ }^{3}$

$$
\boldsymbol{V}=V^{i} \boldsymbol{e}_{i}
$$

with $\boldsymbol{e}_{i}=\partial \boldsymbol{r} / \partial u^{i}, V^{i}=\boldsymbol{V} \cdot \boldsymbol{e}^{i}$ and $\boldsymbol{e}^{i}=\nabla u^{i}$. Now, lets consider a line element $d \boldsymbol{l}$, also in the covariant basis

$$
d \boldsymbol{l}=d u^{i} \boldsymbol{e}_{i}
$$

If the line element $d \boldsymbol{l}$ is always parallel to the magnetic field, then we can write $\boldsymbol{V} \times d \boldsymbol{l}=0$,

\footnotetext{
${ }^{2}$ For near equilibrium plasmas, the projection of the electric field over the magnetic one is small, leading to a small drift across magnetic lines.

${ }^{3}$ Einstein's convention will be used through this text.
} 
or by components

$$
V^{i} d u^{j} \boldsymbol{e}_{i} \times \boldsymbol{e}_{j}=0
$$

using the basic relations between covariant and contravariant basis, $\boldsymbol{e}_{i} \times \boldsymbol{e}_{j}=\sqrt{g} \epsilon_{i j k} \boldsymbol{e}^{k}$, with $\sqrt{g}=\boldsymbol{e}_{i} \cdot\left(\boldsymbol{e}_{j} \times \boldsymbol{e}_{k}\right)$, we get by the linear independence of the contravariant basis

$$
V^{i} d u^{j}-V^{j} d u^{i}=0 \Rightarrow \frac{d u^{i}}{d u^{j}}=\frac{V^{i}}{V^{j}}
$$

Then we are left with a system of differential equations, relating the change in each variable with the changes in the other two, so that the curve is always parallel to the vector field $\boldsymbol{V}$.

\subsubsection{Divergenceless Fields}

From the divergenceless nature of the magnetic field, it can be written as $\boldsymbol{B}=\nabla \times \boldsymbol{A}$ [19], where we are considering $\boldsymbol{A}$ and $\boldsymbol{B}$ as time-independent vector fields in the three dimensional space. In general, the position vector $\boldsymbol{r}$ can be regarded as a function of three independent arbitrary functions $\boldsymbol{r}(\alpha, \beta, \gamma)$, such that each point in the space may be identified uniquely by the three numbers $(\alpha, \beta, \gamma)$. The magnetic potential and in general, any vector may be written

$$
\boldsymbol{A}=A_{\alpha} \nabla \alpha+A_{\beta} \nabla \beta+A_{\gamma} \nabla \gamma
$$

Now, consider the scalar field $\chi(\boldsymbol{r})=\chi(\alpha, \beta, \gamma)$ and its gradient

$$
\nabla \chi=\partial_{\alpha} \chi \nabla \alpha+\partial_{\beta} \chi \nabla \beta+\partial_{\gamma} \chi \nabla \gamma
$$

Equating the $\alpha$-components of $\boldsymbol{A}$ and $\nabla \chi$, we get $A_{\alpha}=\partial_{\alpha} \chi$. Then we can write

$$
A_{\alpha} \nabla \alpha=\nabla \chi-\partial_{\beta} \chi \nabla \beta-\partial_{\gamma} \chi \nabla \gamma
$$

Replacing in the expression for $\boldsymbol{A}$

$$
\boldsymbol{A}=\nabla \chi+\left(A_{\beta}-\partial_{\beta} \chi\right) \nabla \beta+\left(A_{\gamma}-\partial_{\gamma} \chi\right) \nabla \gamma
$$

introducing the functions $\varphi=-A_{\beta}+\partial_{\beta} \chi$, and $\psi=A_{\gamma}-\partial_{\gamma} \chi$ the vector potential may be generally written as

$$
\boldsymbol{A}=\nabla \chi+\psi \nabla \gamma-\varphi \nabla \beta
$$


clearly, the last form is valid for any vector field. Taking the rotational, we have the magnetic field

$$
\boldsymbol{B}=\nabla \psi \times \nabla \gamma-\nabla \varphi \times \nabla \beta
$$

This is also true for any divergenceless vector field.

\subsubsection{Hamiltonian Magnetic Lines}

In (2.17) we have related the magnetic field with the gradients of four scalar fields. Two of them are by hypothesis independent, $\{\beta, \gamma\}$. The remaining fields are quite arbitrary, since they depend on the $\beta$ and $\gamma$ derivatives of some arbitrary scalar field $\chi$ satisfying $A_{\alpha}=\partial_{\alpha} \chi$ only; however we can not say they are always independent from $\beta$ and $\gamma$, since a number of degeneracies may have been introduced through $\chi$.

Now, let us assume that $\{\gamma, \varphi, \beta\}$ are independent functions in several connected domains, those will be established in the particular problem of interest, then or course, we are assuming that $\psi$ is a well defined function of $(\varphi, \beta, \gamma)$ in the interest domain. With this, we can rewrite Eq. (2.17) like

$$
\boldsymbol{B}=\nabla \psi \times \boldsymbol{e}^{\gamma}-\boldsymbol{e}^{\varphi} \times \boldsymbol{e}^{\beta}
$$

Using $\nabla \psi=\partial_{\gamma} \psi \boldsymbol{e}^{\gamma}+\partial_{\varphi} \psi \boldsymbol{e}^{\varphi}+\partial_{\beta} \psi \boldsymbol{e}^{\beta}$, and $\boldsymbol{e}^{i} \times \boldsymbol{e}^{j}=\epsilon_{i j k} \boldsymbol{e}_{k} / \sqrt{g}$ we obtain the magnetic field in the covariant basis.

$$
\sqrt{g} \boldsymbol{B}=-\boldsymbol{e}_{\gamma}+\partial_{\beta} \psi \boldsymbol{e}_{\varphi}-\partial_{\varphi} \psi \boldsymbol{e}_{\beta}
$$

Now we can use Eq. (2.11) to write down the differential equations governing the magnetic tracers or magnetic lines.

$$
\begin{aligned}
\frac{d \beta}{d \gamma} & =\frac{\partial}{\partial \varphi} \psi(\varphi, \beta, \gamma) \\
\frac{d \varphi}{d \gamma} & =-\frac{\partial}{\partial \beta} \psi(\varphi, \beta, \gamma)
\end{aligned}
$$

This are clearly the Hamilton equations, just that the phase space variables and the time, are all spatial variables, which means that this system evolves in two spatial coordinates $(\varphi, \beta)$ as we change the third one $\gamma$, and the orbits will fulfill the conditions imposed by the Hamiltonian mechanics.

Due to arbitrariness of $\beta(\boldsymbol{r})$ and $\gamma(\boldsymbol{r})$, this result is quite general and the meanings of $\psi(\boldsymbol{r})$ and $\varphi(\boldsymbol{r})$ are usually related to different magnetic fluxes, established in the particular geometry of interest. 


\subsection{Hamiltonian Systems}

In the context of Classical Mechanics, the introduction of the Hamiltonian formulation, gives to the problem of motion a geometrical perspective. For a given physical system, it is possible to find a Hamiltonian function $H(p, q, t)$ that characterizes its motion with the dynamical law

$$
\dot{q}_{k}=\frac{\partial H}{\partial p_{k}}, \dot{p}_{k}=-\frac{\partial H}{\partial q_{k}} ;
$$

where $q=\left(q_{1}, \ldots, q_{n}\right)$ and $p=\left(p_{1}, \ldots, p_{n}\right)$, are the generalized coordinates and momenta for a $n$-degrees of freedom system. This vector field drives all solutions in the phase space, and due to its symplectic nature [20], all Hamiltonian systems have similar geometric properties. The power of this formulation goes beyond finding the differential equations that rules a given system; it brings to light important conservation rules that must be followed by the phase flow (integral invariants), and leads to powerful theorems regarding the stability of the motion and robustness against perturbations.

In general, the motions can be classified into regular (fixed, periodic or quasiperiodic) or irregular (wandering/chaotic), depending on the integrability of the system. For a $n$ dimensional integrable system, there is a set of functions $\left\{F_{k}(p, q), k=1, \ldots, n\right\}$ that does not change during the motion (constants of motion), these functions are independent, and must be in involution

$$
\left[F_{\alpha}, F_{\beta}\right]=\sum_{k=1}^{n} \frac{\partial F_{\alpha}}{\partial q_{k}} \frac{\partial F_{\beta}}{\partial p_{k}}-\frac{\partial F_{\beta}}{\partial q_{k}} \frac{\partial F_{\alpha}}{\partial p_{k}}=0
$$

The existence of this set induces the existence of another one, $\left\{I_{k}(p, q), k=1, \ldots, n\right\}$, called action variables and a transformed Hamiltonian $\bar{H}(I)$, that only depends on the actions $I=\left\{I_{1}, \ldots, I_{n}\right\}$. The new Hamiltonian may be obtained through a canonical transformation of the old one [21], and continues to represent the original system, just in a more natural way. Under this transform, the system preserves its Hamiltonian structure and can be integrated directly, since eq. (2.22) is now

$$
\begin{aligned}
& \dot{\theta}_{k}=\frac{\partial \bar{H}}{\partial I_{k}}=\omega_{k}(I), \\
& \dot{I}_{k}=-\frac{\partial \bar{H}}{\partial \theta_{k}}=0,
\end{aligned}
$$

with $\left\{\theta_{k}, k=1, \ldots, n\right\}$, the conjugated coordinates to the new momenta $\left\{I_{k}\right\}$. Clearly, the actions are constant during the motion, or $I_{k}(t)=I_{k}(0)=I_{k}^{0}$ and the angular velocities 
too, with values $\omega_{k}\left(I_{0}\right)$, leading to the complete solution of the problem

$$
\theta_{k}(t)=\theta_{0}+\omega_{k}\left(I_{0}\right) t
$$

However, the existence of the integrals of motion is not generally guaranteed and integrable systems are more the exception, that the rule. The nonexistence of a complete set of integrals of motion leads to wandering or chaotic motion [22], that is quite complex and still not fully understood.

To understand the concept of integrability we can use a simple geometrical approach provided by the Poincaré section. If there are several independent constants, then the orbit must be defined in the intersection of several level sets. When the number of constants of motion equals the number of degrees of freedom, the intersection of all level sets may be mapped to a $n$-dimensional surface or $n$-torus, where the motion is described by $n$-angles changing uniformly. The relation between the frequencies makes the motion periodic or cuasiperiodic, and a single orbit may fill the entire torus if the relation between frequencies is incommensurate. In practice, there is no method to finding constants of motion, so the transformations leading to the action-angle variables are not generally available, and the integrability of the system must be evidenced in other ways.
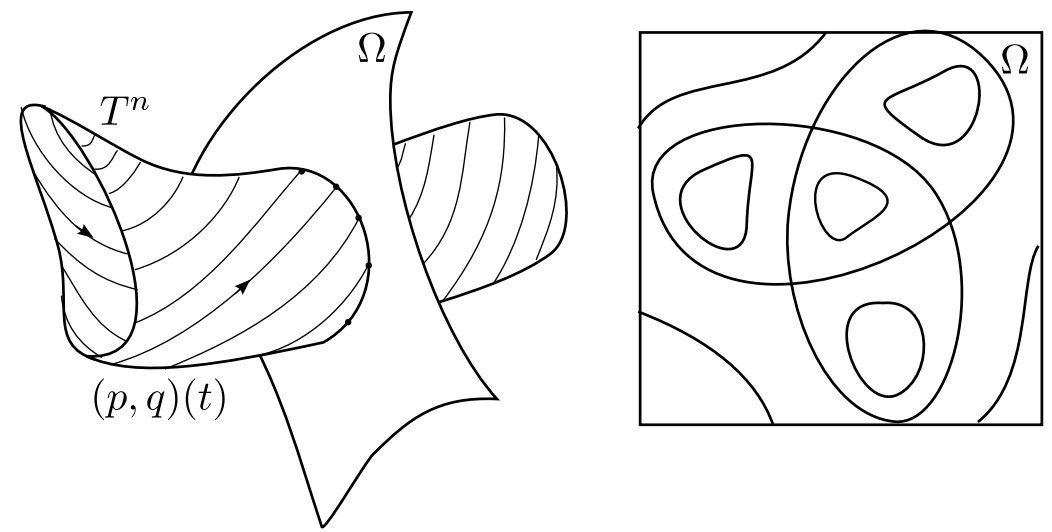

Figure 2.4: Intersection of several trajectories in a torus, with a surface of section.

The Poincaré section consists of a surface in the phase space, that intersects a bunch of orbits of interest. Each intersection in the surface may be considered as an initial condition, now, if the system is bounded, we expect the orbit to return and cross again the surface of section. As each point in the section leads to a single new one, then exist an invertible mapping linking the points over the surface; this is called a Poincaré map, and it possesses most of the dynamical properties of the total system.

When the Hamiltonian is integrable, the orbits rests in a $n$-torus, that intersects the surface in a continuous, $\operatorname{dim}=n-1$ manifold. In the practice we can integrate the equations of motion numerically, and collect the intersections of the orbit with the surface 
of section, then we can see if those points appears to lie in a continuous manifold (fig.2.4). In such case, the Poincaré map moves the points over the manifold and can densely fill it, if the orbit is irrational.
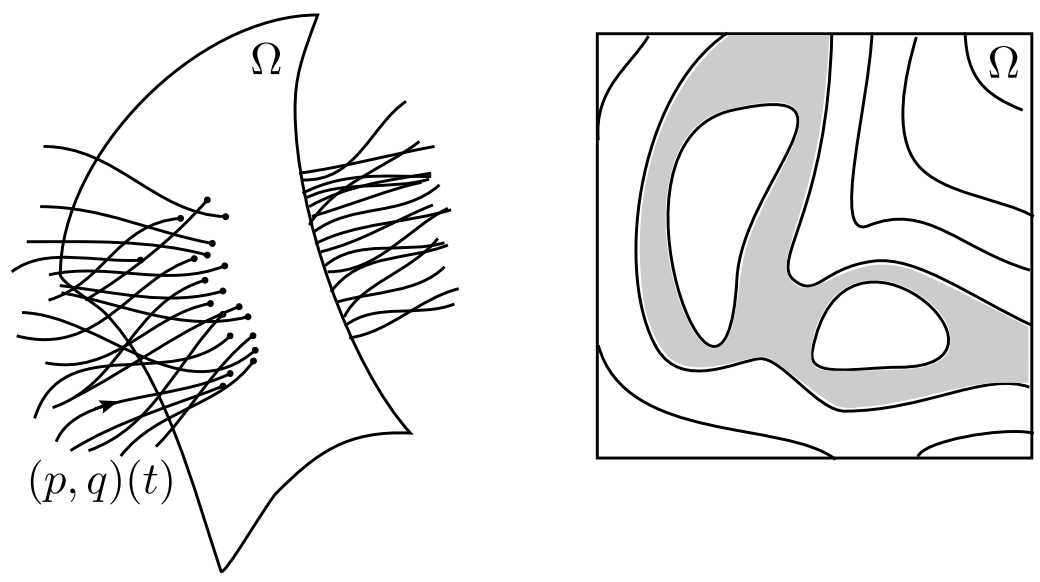

Figure 2.5: Orbits intersection the surface of section, to form an ergodic region.

Chaotic behavior comes from the lack of enough constants of motion. As a consequence the motion can only be restricted to several level sets, but not to a single surface. This means that the orbit is allowed to wander inside a volume in complicated ways and sensitivity to initial conditions appears.

In the Poincaré section of a non-integrable flow, the orbit intersects the surface without being attached to a continuous manifold (Fig. 2.5). This creates ergodic regions where making predictions for a single orbits is ephemeral. Actually, from the recurrence theorem of Poincaré [20], ergodic orbits must fill the volume where they are defined, therefore a statistical description seems to be more appropriate.

A way to better understand chaotic behavior is by introducing quasi-integrable systems, usually composed from a principal integrable Hamiltonian and a small, timedependent, perturbing potential [23]. The size of the perturbation allows to control the effect on the topology of the integrable system. According to the Kolmogorov-ArnoldMoser (KAM) theorem [20], it is possible to show that for a small intensity, there is no full destruction of the regular surfaces; but all the rational ones ${ }^{4}$ are destroyed. By the nature of rational numbers, the phase space is layered by chaotic regions, with invariant surfaces, between them. The increase of the perturbation leads to further destruction of invariant surfaces, starting about the separatrixes that connects two hyperbolic points (heteroclinic orbits), or an hyperbolic point to itself (homoclinic orbits). The PoincaréBirkhoff theorem for fixed points [24], ensures that under a perturbation, points are born in pairs (elliptic-hyperbolic), at the resonant surfaces, where the unperturbed frequencies

\footnotetext{
${ }^{4}$ Those with commensurate frequencies respect to the perturbation
} 
and the perturbation frequency are commensurate, this is why all rational surfaces are destroyed by the perturbation. 


\section{Chapter 3}

\section{MHD EQUILIBRIUM}

Under suitable approximations the plasma can be described as a single fluid, governed by the Magnetohydrodinamic (MHD) model [2]. To derive this model we can combine the statistical descriptions of the species in the plasma with a description of the fields produced by their charges and their motion. If we consider the phase space distribution function describing the state of the plasma specie $\alpha$ for a given time, $f_{\alpha}(\boldsymbol{r}, \boldsymbol{u}, t)$, we can couple the Boltzmann equation to the Maxwell equations for the electromagnetic field

$$
\begin{aligned}
& \frac{\partial f_{\alpha}}{\partial t}+\boldsymbol{u} \cdot \nabla f_{\alpha}+\frac{q_{\alpha}}{m_{\alpha}}(\boldsymbol{E}+\boldsymbol{u} \times \boldsymbol{B}) \cdot \nabla_{u} f_{\alpha}=\left[\frac{\delta f_{\alpha}}{\delta t}\right]_{\text {coll }}, \\
& \nabla \times \boldsymbol{E}=-\frac{\partial B}{\partial t}, \\
& \nabla \cdot \boldsymbol{B}=0, \\
& \nabla \times \boldsymbol{B}=\mu_{0} \boldsymbol{j}+\frac{1}{c^{2}} \frac{\partial \boldsymbol{E}}{\partial t}=\frac{1}{c^{2}} \frac{\partial \boldsymbol{E}}{\partial t}+\mu_{0} \sum_{\alpha} q_{\alpha} \int \boldsymbol{u} f_{\alpha} d \boldsymbol{u}, \\
& \nabla \cdot \boldsymbol{E}=\frac{\sigma}{\varepsilon_{0}}=\frac{1}{\varepsilon_{0}} \sum_{\alpha} q_{\alpha} \int f_{\alpha} d \boldsymbol{u} .
\end{aligned}
$$

Here, the electric and magnetic fields are produced by the mean distributions of current and charge density inside the plasma, and those fields affect back the distributions $f_{\alpha}$ through the forces they exert on the particles. Although complete, this model is exceedingly complex, and analytical, even numerical approximations are impossible in general cases.

Lets start by considering that the plasma is constituted by electrons and ions only $(\alpha=$ $e, i$ ), and that ions are once charged (i.e. their charge is $e$ ). Also, instead of considering the Boltzmann distribution $f_{\alpha}$, we can consider the moments of the distribution, obtained by averaging in the velocity space the quantities $Q_{1}=1, Q_{2}=m_{\alpha}, Q_{3}=\frac{1}{2} m_{\alpha} u^{2}$, related to the density of particles, momentum and energy in a given point of the space. Taking into consideration the usual contributions to the distribution function by collisions between 
particles of the same and different species, together with the conservation of momentum, energy and particles, and defining the means

$$
\begin{array}{r}
n_{\alpha} \equiv \int f_{\alpha} d \boldsymbol{u}, \\
\boldsymbol{v}_{\alpha} \equiv \frac{1}{n_{\alpha}} \int \boldsymbol{u} f_{\alpha} d \boldsymbol{u},
\end{array}
$$

we obtain the set of two-fluid equations.

$$
\begin{aligned}
& \frac{d n_{\alpha}}{d t}+n_{\alpha} \nabla \cdot \boldsymbol{v}_{\alpha}=0, \\
& n_{\alpha} m_{\alpha} \frac{d \boldsymbol{v}_{\alpha}}{d t}-q_{\alpha} n_{\alpha}\left(\boldsymbol{E}+\boldsymbol{v}_{\alpha} \times \boldsymbol{B}\right)+\nabla \cdot \mathbb{P}_{\alpha}=\boldsymbol{R}_{\alpha}, \\
& \frac{3}{2} n \frac{d T_{\alpha}}{d t}+\mathbb{P}_{\alpha}: \nabla \boldsymbol{v}_{\alpha}+\nabla \cdot \boldsymbol{h}_{\alpha}=Q_{\alpha}, \\
& \nabla \times \boldsymbol{E}=-\frac{\partial \boldsymbol{B}}{\partial t}, \\
& \nabla \times \boldsymbol{B}=\mu_{0} e\left(n_{i} \boldsymbol{v}_{i}-n_{e} \boldsymbol{v}_{e}\right)+\frac{1}{c^{2}} \frac{\partial \boldsymbol{E}}{\partial t} \\
& \nabla \cdot \boldsymbol{E}=\frac{e}{\varepsilon_{0}}\left(n_{i}-n_{e}\right), \\
& \nabla \cdot \boldsymbol{B}=0 .
\end{aligned}
$$

Where the temperature, scalar and tensorial pressure were defined like

$$
p_{\alpha} \equiv \frac{1}{3} n_{\alpha} m_{\alpha}<w_{\alpha}^{2}>, \mathbb{P}_{\alpha} \equiv n_{\alpha} m_{\alpha}<\boldsymbol{w}_{\alpha} \boldsymbol{w}_{\alpha}>, T_{\alpha} \equiv \frac{p_{\alpha}}{n_{\alpha}},
$$

with $\boldsymbol{w}_{\alpha}=\boldsymbol{u}-\boldsymbol{v}_{\alpha}$ a relative velocity and $<>$ the velocity space mean. In the equations also appears the quantities $\boldsymbol{R}_{\alpha}, Q_{\alpha}, \boldsymbol{h}_{\alpha}$, related to, the mean momentum transfer between unlike particles due to collisions, the heat produced in such events, and the heat flux by random motions (details in [2]), respectively.

\subsection{Single Fluid Equations}

The most tractable model of a plasma, corresponds to that of a single fluid, whose properties may be averaged from the two fluid model. To obtain this description, a number of considerations are in order. First, the plasma is considered to be locally quasi-neutral $\left(n_{i}=n_{e}=n\right)$ and the displacement current is neglected $\left(\varepsilon_{0} \partial \boldsymbol{E} / \partial t\right)$, which amounts to consider waves with velocities much less that light speed. Also, the neutrality may be understood as doing $\varepsilon_{0} \nabla \cdot \boldsymbol{E}=0$, valid for frequencies much less that plasma frequency $\omega_{p e} \equiv \sqrt{n e^{2} / m_{e} \varepsilon_{0}}$, and lengths much larger that the Debye length $\lambda_{d}=v_{T e} / \omega_{p e}\left(v_{t e}\right.$ is 
the electronic thermal velocity). The inertia of the electron is also neglected $m_{e} \rightarrow 0$, in comparison with that of the ions ( $\sim 4000$ times larger). Under this approximations, and the following definitions

$$
\begin{aligned}
& \rho=m_{i} n, \\
& \boldsymbol{v}=\boldsymbol{v}_{i}, \\
& \boldsymbol{J}=e n\left(\boldsymbol{v}_{i}-\boldsymbol{v}_{e}\right), \\
& p=n T=p_{e}+p_{i}, \\
& T=T_{e}+T_{i}
\end{aligned}
$$

a the single fluid equations are found, and after another number of approximations [2], related to the collisionality of the plasma, and the spatial scale of interest we get:

$$
\begin{aligned}
& \frac{\partial \rho}{\partial t}+\nabla \cdot \rho \boldsymbol{v}=0, \\
& \rho \frac{d \boldsymbol{v}}{d t}=\boldsymbol{j} \times \boldsymbol{B}-\nabla p, \\
& \frac{d}{d t} \frac{p}{\rho^{\gamma}}=0 \\
& \boldsymbol{E}+\boldsymbol{v} \times \boldsymbol{B}=0 \\
& \nabla \times \boldsymbol{E}+\frac{\partial \boldsymbol{B}}{\partial t}=0 \\
& \nabla \times \boldsymbol{B}-\mu_{0} \boldsymbol{j}=0, \\
& \nabla \cdot \boldsymbol{B}=0
\end{aligned}
$$

where the time derivative is a convective derivative, or $d / d t=\partial / \partial t+\boldsymbol{v} \cdot \nabla$, to be taken in the fluid's reference frame. This set of equations is valid for a long-wavelength, lowfrequency, dynamics in a macroscopic plasma.

\subsection{Equilibrium}

In Dynamical Systems theory, even when the main concern is about dynamics, the evolution of the system is strongly affected by the types of equilibria that can be achieved. The search for fixed points is important, because the evolution of sufficiently near conditions can be described by a linear system, and time-dependent solutions adjust themselves to satisfy the local conditions imposed about equilibrium configurations.

As an example, in Fig.3.1, there is a representation of the local linear dynamics in a two-dimensional dynamical system. Assuming we have four kind of fixed points: a center (a), two saddles (b,c), a spiral source (c) and a spiral sink (e), and knowing the local 
dynamics about them, we can construct a good model of the global flow of solutions. The local manifolds of the fixed points restricts some of the global topology, of course depending on the relative positions of those equilibria in the phase space, and on the stability of each one.

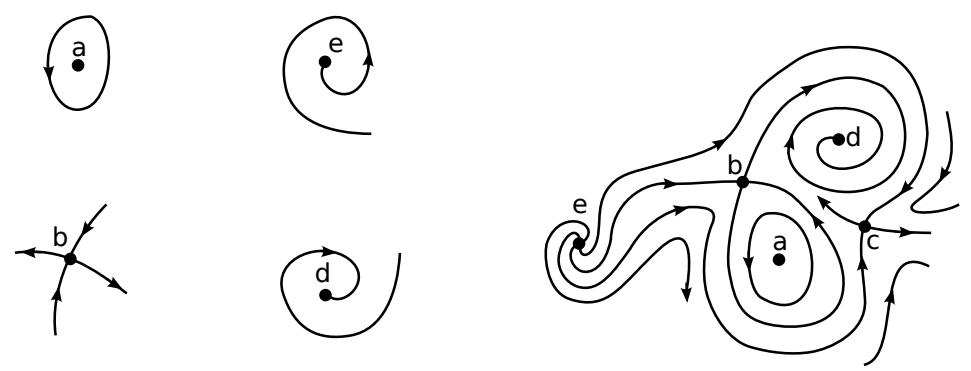

Figure 3.1: Fixed points and their relation to the global flux.

In the context of plasmas, there is a set of functions $(\rho, p, \boldsymbol{B}, \boldsymbol{E}, \boldsymbol{j}, \boldsymbol{v})$, depending on the position $\boldsymbol{r}$ and time $t$, that describes the plasma state. We also have a set of equations (eqs.(3.21)-(3.27)), that defines the change on each variable as a function of the others, i.e. the system's dynamics. This is a more general dynamical system, where the state variable is the set of functions describing the plasma at each point in the space. We can expect that a local linear analysis about an equilibrium configuration may give important information about the stability of the system in near configurations. Despite the clear differences between this dynamical system and the most elementary ones (described by a countable set of state variables), we can start the analysis of the plasma with its equilibrium configurations.

The single fluid equations describe only "slow" phenomena in a "smooth" plasma, but still a very good model for macroscopic plasmas, even going beyond its scope of application; using the fluid equations, we can assume the existence of an equilibrium configurations of the plasma. This amounts to make $\partial / \partial t=0$, in all quantities, and asking for $\boldsymbol{v}=0$, so that the convective derivative vanishes, leading to the reduced set of equations

$$
\begin{aligned}
& \nabla p=\boldsymbol{j} \times \boldsymbol{B}, \\
& \nabla \times \boldsymbol{B}=\mu_{0} \boldsymbol{j}, \\
& \nabla \cdot \boldsymbol{B}=0 .
\end{aligned}
$$

Any equilibrium configuration of the plasma must satisfy this set of equations. Although this system is much simpler that the dynamical one, its solution is quite difficult, and still a serious issue on the problem of confinement. Before constructing the formal description of equilibrium, we can make some elementary observations. 

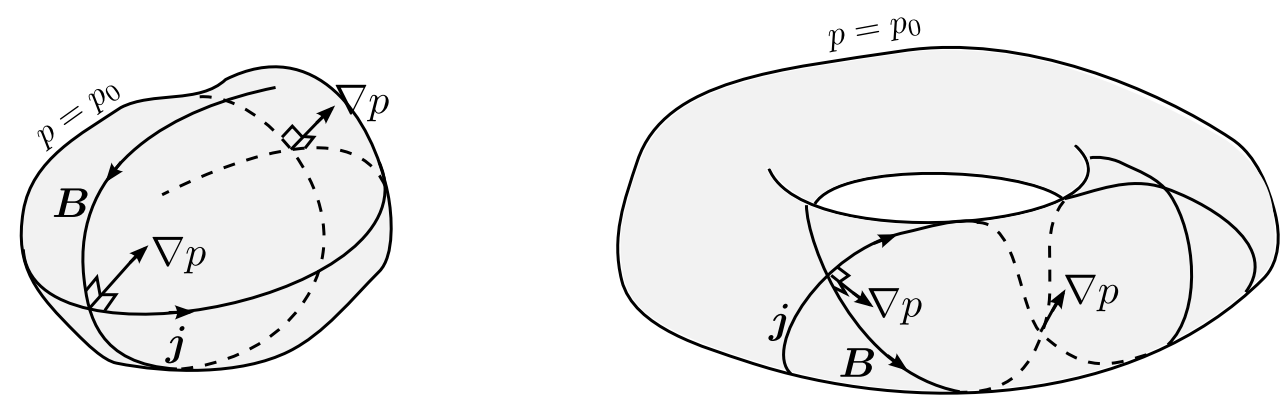

Figure 3.2: Simply connected isobaric surfaces violate the equilibrium condition (3.28).

Assume we manage to confine a given volume of plasma in a finite region of space. From the macroscopic point of view, the kinetic pressure is a smooth function, then the equation $p=$ const. defines a smooth isobaric surface in the space. If the plasma is actually confined, we require the $p=$ const. surfaces to be closed. From eq. (3.28), the current and magnetic field lines clearly extends over an isobaric surface, so that $\boldsymbol{j} \times \boldsymbol{B}$ is always normal to it. However this introduces a restriction on the shape of the isobaric surfaces, that can not be simply connected (see fig.3.2). If the isobaric surface is simply connected, and simultaneously satisfies (3.28), then two crossing stream lines define a direction normal to the surface, but if they cross again as they move over the surface, the normal direction will be reversed introducing a contradiction. This problem is solved if we consider that the isobaric surfaces are not simply connected, and the simplest example of such geometry is the torus $T^{2}$; this is another verification of the basic statements made in previous sections, about the minimal model allowing to confine plasmas. In the right of fig. 3.2 we can see that the magnetic and current stream lines always cross in a way compatible with the surface orientation.

\subsubsection{Grad-Shafranov Equation}

In the following, the last set of equations will be summarized in a single well known equation describing the plasma equilibria for any axisymmetric system. To do this, we have to adopt a convenient representation of the quantities, here, cylindrical coordinates $(R, \phi, z)$ are adequate. In this representation, axisymmetry means that all quantities are independent of the toroidal angle $\phi$. From eq. (3.30) we can introduce a vector potential $\boldsymbol{A}$ such that, $\boldsymbol{B}=\nabla \times \boldsymbol{A}$, where $\partial A_{\alpha} / \partial \phi=0$ and $\alpha=R, \phi, z$. Then, in cylindrical coordinates we have

$$
\boldsymbol{B}=-\hat{\boldsymbol{R}} \partial_{z} A_{\phi}+\hat{\boldsymbol{\phi}}\left(\partial_{z} A_{R}-\partial_{R} A_{z}\right)+\hat{z} R^{-1} \partial_{R}\left(R A_{\phi}\right)
$$


Defining $B_{\phi}(R, z)=\partial_{z} A_{R}-\partial_{R} A_{z}$, and reordering we get

$$
\boldsymbol{B}=\left[\hat{\boldsymbol{z}} R \partial_{z} A_{\phi}+\hat{\boldsymbol{R}} \partial_{R}\left(R A_{\phi}\right)\right] \times\left(R^{-1} \hat{\boldsymbol{\phi}}\right)+\hat{\boldsymbol{\phi}} B_{\phi}
$$

using the gradient in cylindrical coordinates, noting that $\nabla \phi=R^{-1} \hat{\boldsymbol{\phi}}$, and defining $\psi(R, z)=R A_{\phi}(R, z)$, we have

$$
\boldsymbol{B}=\nabla \psi \times \nabla \phi+\hat{\boldsymbol{\phi}} B_{\phi}
$$

This type of representation was already expected, but here takes a more specific form in terms of known coordinates and quantities. Before keep going, lets give a physical meaning to the quantity $\psi$. Recall that $\boldsymbol{B}=\nabla \times \boldsymbol{A}$, then, the magnetic flux through a given surface $\sigma$, limited by a closed curve $\delta \sigma$, is by the Stokes theorem:

$$
\Phi_{B}(\sigma)=\int_{\sigma} \boldsymbol{B} \cdot d \boldsymbol{S}=\int_{\delta \sigma} \boldsymbol{A} \cdot d \boldsymbol{l}
$$

Considering a boundary $\delta \sigma$, whose line element has only toroidal component, $(d \boldsymbol{l}=d l \hat{\boldsymbol{\phi}}=$ $R d \phi \hat{\boldsymbol{\phi}})$, and using $\psi=R A_{\phi}$, we have

$$
\Phi_{B}(\sigma)=\int_{\delta \sigma} \psi d \phi=2 \pi \psi
$$

where was considered that $\psi$ is axisymmetric. Then $\psi$ is proportional to the magnetic poloidal flux through a surface limited by boundaries with constant $R$ and $z$.

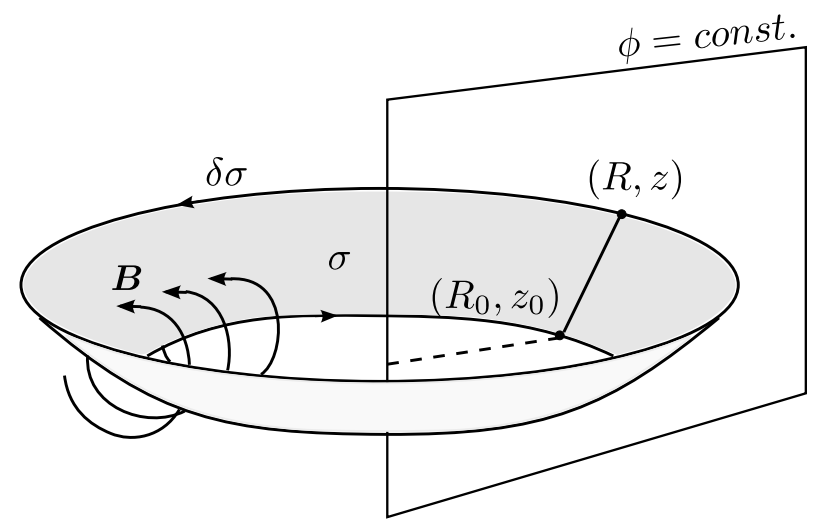

Figure 3.3: Surface to calculate the poloidal magnetic flux, and the poloidal current.

In fig.3.3 we can see the basic shape of the surface used to calculate the magnetic flux. As the magnetic field is expected to rest in a toroidal surface, then the surface $\sigma$ must be limited by two closed curves, so that the net flux of magnetic field is not zero. One of the loops is kept fixed at $\left(R_{0}, z_{0}\right)$, where we usually have a magnetic axis, i.e. a purely 
toroidal magnetic field.

As the current density $\boldsymbol{j}$, can be written, $\boldsymbol{j}=\mu_{0}^{-1} \nabla \times \boldsymbol{B}$, we can apply the same reasoning and write

$$
\mu_{0} \boldsymbol{j}=-\hat{\boldsymbol{R}} \partial_{z} B_{\phi}+\hat{\boldsymbol{\phi}}\left(\partial_{z} B_{R}-\partial_{R} B_{z}\right)+\hat{\boldsymbol{z}} R^{-1} \partial_{R}\left(R B_{\phi}\right)
$$

The toroidal current is clearly $\mu_{0} j_{\phi}(R, z)=\partial_{z} B_{R}-\partial_{R} B_{z}$, reordering we have

$$
\boldsymbol{j}=\mu_{0}^{-1}\left[\hat{\boldsymbol{z}} R \partial_{z} B_{\phi}+\hat{\boldsymbol{R}} \partial_{R}\left(R B_{\phi}\right)\right] \times\left(R^{-1} \hat{\boldsymbol{\phi}}\right)+\hat{\boldsymbol{\phi}} j_{\phi}
$$

now we introduce the quantity $F(R, z)=R B_{\phi}(R, z)$, to get

$$
\boldsymbol{j}=\mu_{0}^{-1} \nabla F \times \nabla \phi+\hat{\boldsymbol{\phi}} j_{\phi}
$$

Here, $F$ has the meaning of the poloidal current density flux, or poloidal current, through a surface like in fig.3.3. The topology of the plasma is defined by the family of surfaces $\psi=$ const. Clearly we expect those surfaces to be closed although not simply connected.

In general, any vector field may be decomposed in poloidal and toroidal components, where the poloidal direction lies on a plane $\phi=$ const . and therefore is perpendicular to the toroidal one; with this, the force balance equation (3.28) is written

$$
\nabla p=\left(\boldsymbol{j}_{p}+\boldsymbol{j}_{\phi}\right) \times\left(\boldsymbol{B}_{p}+\boldsymbol{B}_{\phi}\right)
$$

As we are dealing with a axisymmetric configuration, the pressure is $\phi$-independent, then $\nabla p$ lies also in a $\phi=$ const. plane and the previous equation may be decomposed into

$$
\begin{aligned}
& \boldsymbol{j}_{p} \times \boldsymbol{B}_{p}=0, \\
& \nabla p=\boldsymbol{j}_{p} \times \boldsymbol{B}_{\phi}+\boldsymbol{j}_{\phi} \times \boldsymbol{B}_{p} .
\end{aligned}
$$

Then the poloidal components of the magnetic field and the current density are parallel. Identifying the poloidal components in (3.33) and (3.38) as $\boldsymbol{B}_{p}=\nabla \psi \times \nabla \phi$ and $\boldsymbol{j}_{p}=$ $\mu_{0}^{-1} \nabla F \times \nabla \phi$; then using the identity $(a \times b) \times(c \times d)=(a \times b \cdot d) c-(a \times b \cdot c) d$, we get

$$
\nabla F \times \nabla \phi \cdot \nabla \psi=0 \Rightarrow \nabla \psi \times \nabla F \cdot \nabla \phi=0
$$

as $\psi$ and $F$, are $\phi$-independent, their gradients rest in the $\phi=$ const. plane, so $\nabla \phi \times \nabla F$ must be in direction $\phi$, then the eq.(3.42) means

$$
\nabla \psi \times \nabla F=0 \Rightarrow \nabla \psi \| \nabla F
$$


As $\nabla \psi$ is always orthogonal to any $\psi=$ const. surface, then $\nabla F$ is too; but $\nabla F$ is orthogonal to the $F=$ const. surfaces. This implies that the surfaces $\psi=c_{1}$ and $F=c_{2}$ are equal. For each surface there is a unique value of $\psi$ and a unique value of $F$, and both change continuously from surface to surface, then, exists a continuous function relating them and we can write

$$
F(R, z)=F(\psi(R, z))
$$

This means that $F$ is a surface quantity.

Now, replacing the poloidal components of $\boldsymbol{B}$ and $\boldsymbol{j}$ into (3.41), using the relation $a \times(b \times c)=(a \cdot c) b-(a \cdot b) c$, we have

$$
R \nabla p=j_{\phi} \nabla \psi-\mu_{0}^{-1} B_{\phi} \nabla F
$$

from (3.44) we have $\nabla F=(d F / d \psi) \nabla \psi$, so we have

$$
\nabla p=R^{-1}\left(j_{\phi}-\mu_{0}^{-1} B_{\phi} F^{\prime}\right) \nabla \psi
$$

This equation indicates that $\nabla p$ is always parallel to $\nabla \psi$, then $p$ is also a surface quantity. In a toroidal confinement scheme, we expect the isobaric surfaces to be toroidal, then the flux surfaces will be toroidal as well. We can write $\nabla p=(d p / d \psi) \nabla \psi$, then for $\nabla \psi \neq 0$, (3.46) reduces to

$$
j_{\phi}=R \frac{d p}{d \psi}+\mu_{0}^{-1} R^{-1} F \frac{d F}{d \psi}
$$

Then, to know the toroidal current density we need to know the functions $F(\psi)$ and $p(\psi)$; but those are not generally available, and their form depends on the equilibrium. The choice of this functions is made so as to simplify the analytical treatment of the system, but they are mathematically arbitrary, and physically they must guarantee the self-consistency of the solution.

To finalize the derivation of the Grad-Shafranov (G-S) equation, lets use the Ampere's law (3.29), in terms of the magnetic potential $\boldsymbol{A}$. Then we have

$$
\mu_{0} \boldsymbol{j}=\nabla \times(\nabla \times \boldsymbol{A})=\nabla(\nabla \cdot \boldsymbol{A})-\nabla^{2} \boldsymbol{A}
$$

In the Coulomb gauge $\nabla \cdot \boldsymbol{A}=0$, then $\nabla^{2} \boldsymbol{A}=-\mu_{0} \boldsymbol{j}$; then we can take the $\phi$-component of this equation, to use the representation of $j_{\phi}$ in (3.47). In cylindrical coordinates we have $\left(\nabla^{2} \boldsymbol{A}\right)_{\phi}=\nabla^{2} A_{\phi}+2 R^{-2} \partial_{\phi} A_{R}-R^{-2} A_{\phi}$; from axisymmetry we have $\partial_{\phi}()=0$, and the $\phi$-component of eq. (3.48) is

$$
-\mu_{0} j_{\phi}=\nabla^{2} A_{\phi}-R^{-2} A_{\phi} .
$$


Expanding the laplacian as, $\nabla^{2} A_{\phi}=R^{-1} \partial_{R}\left(R \partial A_{\phi}\right)+\partial_{z}^{2} A_{\phi}$, and replacing $A_{\phi}=\psi / R$, it is easy to show that

$$
R \nabla \cdot\left(R^{-2} \nabla \psi\right)=-\mu_{0} j_{\phi}
$$

so, after replacing (3.47), we obtain the Grad-Shafranov equation:

$$
-R^{2} \nabla \cdot\left(R^{-2} \nabla \psi\right)=\mu_{0} R^{2} \frac{d p}{d \psi}+F \frac{d F}{d \psi}
$$

or

$$
-\left(\partial_{R}^{2} \psi-R^{-1} \partial_{R} \psi+\partial_{z}^{2} \psi\right)=\mu_{0} R^{2} \frac{d p}{d \psi}+F \frac{d F}{d \psi} .
$$

It is also customary, to define the elliptic operator $\Delta^{*}()=R^{2} \nabla \cdot\left(R^{-2} \nabla()\right)$.

The G-S equation, is a second order elliptic partial differential equation. It also may be linear or nonlinear, depending on the choice of the source functions $p(\psi), F(\psi)$. As the non-homogeneity $j_{\phi}$ depends intrinsically on the solution $\psi(R, z)$, the flux $\psi$ is both an independent and dependent variable, since the problem is nonlinear it must be solved self-consistently to match the boundary conditions. Another difficulty arises, because there is no way to establish the edge of the plasma before actually solving the problem, in general the boundary conditions are not available, making this, a free boundary problem.

Albeit all the problems establishing a proper set of conditions for the poloidal flux $\psi(R, z)$, we expect the solutions of the G-S equation to be smooth, so that the magnetic field lines wind kindly over the level surfaces $\psi=$ const. This is a consequence of asking for axisymmetry, that automatically makes the Hamiltonian of the magnetic lines an integrable one, and the phase flow (eqs. (2.20-2.21)) becomes stationary respect to $\phi$. In fact, without axisymmetry we must solve the full 3-dimensional problem, where the magnetic surfaces existence is not guaranteed, and the problem is in general non-integrable. A great advantage of assuming axisymmetry is that we can introduce the asymmetric effects as a small perturbation that destroys some of the magnetic surfaces, but leads some invariant surfaces where the magnetic field still integrable, and we can study the transition from laminar fields to ergodic ones.

For any Hamiltonian bounded system, depending just on two variables, there are local transformations leading to action-angle formalism. In other words, we expect the magnetic surfaces to be smooth and nested, at least locally. Then the equilibria from the G-S equation must consist in general of sets of nested surfaces with axial symmetry, but there is no formal restriction on the total number of magnetic axis (fig.3.4). 


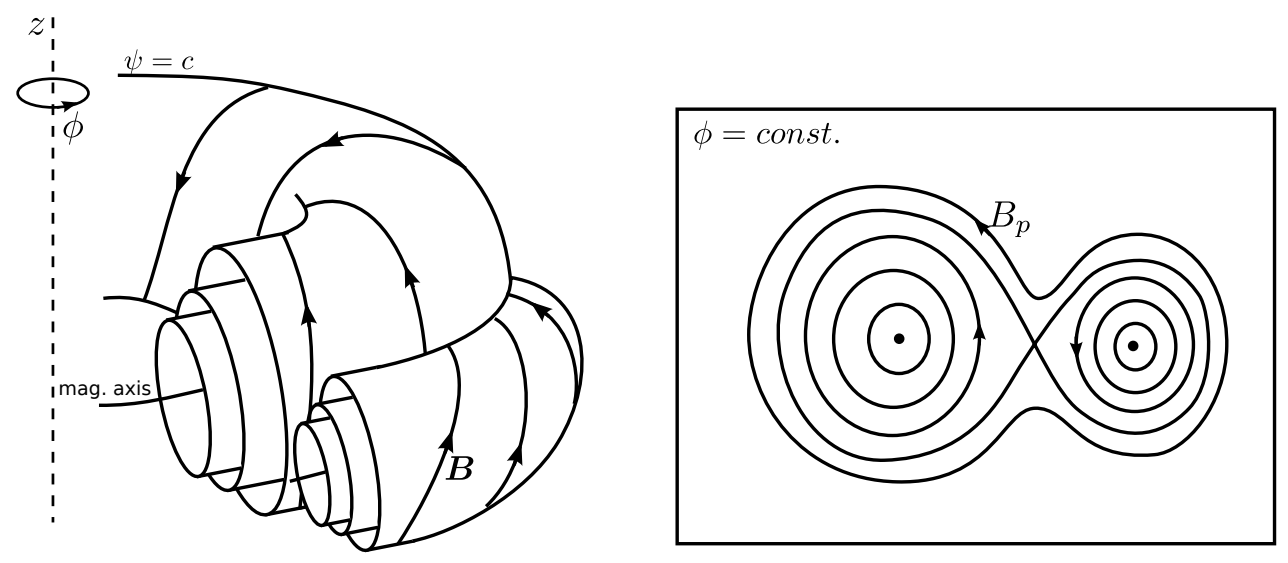

Figure 3.4: The equilibrium field lines wind on sets of nested magnetic surfaces about a finite number of magnetic axes.

\subsection{Equilibrium Solutions}

Given the arbitrariness of the source functions $F(\psi)$ and $p(\psi)$, there are several ways to solve the Grad-Shafranov equation by choosing particular forms of the toroidal current $j_{\phi}$. Some choices of $F(\psi)$ and $p(\psi)$ lead to linear G-S equations, then, provided a suitable set of boundary conditions, we can obtain analytical solutions that may be used to describe experimental results $[25,26]$. From Sturm-Liouville theory [27], we know that a linear differential operator, has associated a full set of eigenfunctions, that may be used to expand any general solution matching the boundary conditions and obeying the differential equation containing the operator; then it is expected that, for separable G-S equations, linear solutions model accurately the plasma shape, while their most serious limitation is in describing the kinetic pressure, and toroidal magnetic field, that usually have oversimplified dependences on the magnetic flux $\psi$. Clearly, choosing a more accurate form of the "arbitrary" functions, lead to nonlinearity of the G-S equation, where analytical solutions are not generally available and numerical methods must be used to solve for general boundary conditions.

\subsubsection{Boundary Conditions}

Before introducing the successive approximation method lets review the usual boundary conditions for plasmas confined inside conducting chambers. First of all, a vacuum magnetic field is needed to confine the plasma. If successful confinement is reached, the plasma occupies a finite volume in space (that we expect to be toroidal), and in equilibrium it has a well defined edge (free boundary). There is also a toroidal conducting chamber containing the plasma, that helps to balance the outward hoop forces produced by the current loop inside the plasma [2]. The poloidal magnetic flux $\psi$ is defined inside the 
plasma region, and $\psi_{v}$ in the vacuum between the plasma and the conducting wall (fig. $3.5)$.

In the most simple case, we have a circular cross section chamber. As magnetic fields are normal to perfectly conducting surfaces, the poloidal field in the surface $\sigma_{2}$ must vanish. In terms of the poloidal flux, $\boldsymbol{B}_{p}=\nabla \psi \times \nabla \phi$, then we have $\partial \psi_{v} / \partial n_{2}=0$, with $\hat{n}_{2}$ the normal direction to $\sigma_{2}$.

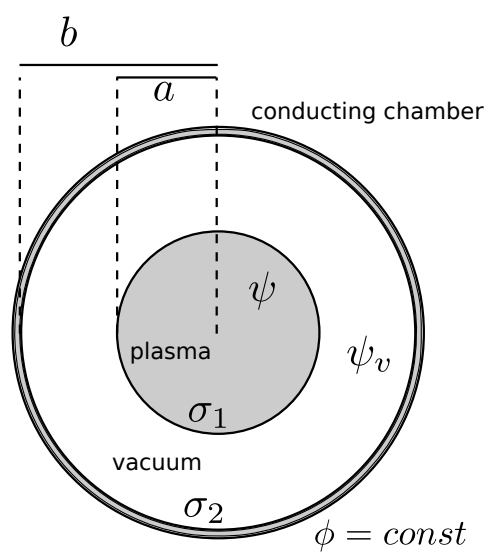

Figure 3.5: Regions of interest and boundaries.

As in the vacuum there are not moving (or still) charges, the current density vanishes and the G-S equation is reduced to $\Delta^{*} \psi_{v}=0$. Considering only the magnetic field produced by the plasma, it is clear that the conducting shell must be over a magnetic surface; and by continuity of $\psi_{v}$ the vacuum magnetic surfaces must be a set of nested tori. As $\psi$ has the meaning of a flux, it must be continuous, then we can match $\psi\left(\sigma_{1}\right)=$ $\psi_{v}\left(\sigma_{1}\right)$. Also, an induced current in the plasma surface leads to a discontinuity on the magnetic field given by $\hat{n}_{1} \times\left(\boldsymbol{B}_{\text {out }}-\boldsymbol{B}_{\text {in }}\right)=\mu_{0} \boldsymbol{j}_{s}$; the toroidal component of this is, $\partial \psi_{v} / \partial n_{1}-\partial \psi / \partial n_{1}=j_{\phi}\left(\sigma_{1}\right)$. The toroidal current may be written in terms of the change in the arbitrary functions $F(\psi)$ and $p(\psi)$. If we allow the pressure to vanish smoothly at the plasma edge, and consider that $F$ does not change there, since there is no contribution to the poloidal current in the vacuum; then $j_{\phi}\left(\sigma_{1}\right)=0$ and $\partial \psi_{v} / \partial n_{1}=\partial \psi / \partial n_{1}$. When we allow discontinuities on the pressure at the edge, we also introduce discontinuities in the magnetic field. From equilibrium we have

$$
\nabla p=\mu_{0}^{-1}(\nabla \times \boldsymbol{B}) \times \boldsymbol{B}=\mu_{0}^{-1} \boldsymbol{B} \cdot \nabla \boldsymbol{B}-\mu_{0}^{-1} \nabla\left(B^{2} / 2\right) .
$$

Taking a dot product with a differential vector $\delta \boldsymbol{r}$ normal to the magnetic surfaces (then to the magnetic field), we have

$$
\nabla\left(p+\frac{B^{2}}{2 \mu_{0}}\right) \cdot \delta \boldsymbol{r}=\delta\left(p+\frac{B^{2}}{2 \mu_{0}}\right)=0
$$


where $\delta \alpha$, represents the change in $\alpha$, after an infinitesimal displacement $\delta \boldsymbol{r}$. This must also hold at the boundary $\sigma_{1}$, then can be used as a boundary condition.

\subsubsection{Successive Approximations}

Now, lets introduce a successive approximation method, valid for large aspect ratio tokamaks and generic choices of source functions. Lets write the G-S equation in local polar coordinates (fig. 3.5). This coordinates are defined by the transformations

$$
R=R_{0}+r \cos \theta, z=r \sin \theta, \phi=\phi
$$

clearly, the transformation is one to one only if $r<R_{0}$, which is sufficient to describe the equilibrium in tokamaks.

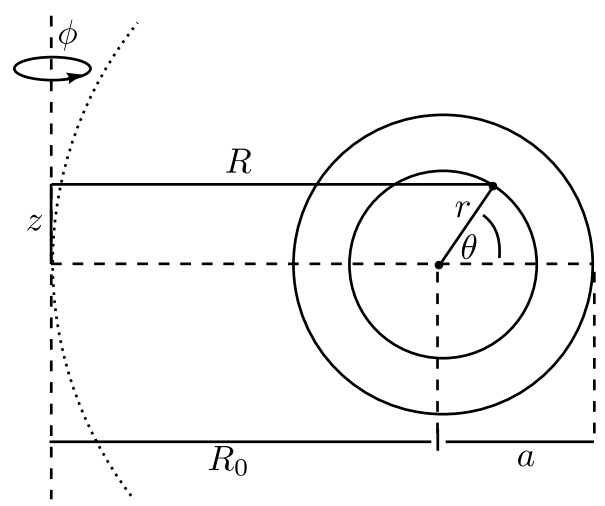

Figure 3.6: Local polar coordinate system.

In the elliptic operator $\Delta^{*}=\partial_{R}^{2}+\partial_{z}^{2}-R^{-1} \partial_{R}$, the first terms $\partial_{R}^{2}+\partial_{z}^{2}$ act like the Laplace operator $\nabla^{2}$ in a plane. The action of $\nabla^{2}$ does not change by changing the origin, then we can write $\Delta^{*}=\nabla^{2}-R^{-1} \partial_{R}$ where $R$ must be written in local polar coordinates. It can be shown that $\partial r / \partial R=\cos \theta$ and $\partial \theta / \partial R=-\sin \theta / r$, then replacing $R$ and $\partial / \partial R$ in $\Delta^{*}$ we get

$$
\Delta^{*}=\nabla^{2}-\frac{1}{R_{0}+r \cos \theta}\left(\cos \theta \frac{\partial}{\partial r}-\frac{\sin \theta}{r} \frac{\partial}{\partial \theta}\right) .
$$

Let us assume that the plasma edge is about the circle $r=a$, introducing the non dimensional radius $\tilde{r}$ defined by $r=a \tilde{r}$, the G-S operator may be written $\Delta^{*}=a^{-2} \tilde{\Delta}^{*}$, where

$$
\tilde{\Delta}^{*}=\tilde{\nabla}^{2}-\frac{\epsilon}{1+\tilde{r} \epsilon \cos \theta}\left(\cos \theta \frac{\partial}{\partial \tilde{r}}-\frac{\sin \theta}{\tilde{r}} \frac{\partial}{\partial \theta}\right) .
$$

Here $\tilde{\nabla}^{2}$ is a non dimensional laplacian, and $\epsilon=a / R_{0}$ is a geometric property of equilibrium called inverse aspect ratio. We can also write the poloidal flux in terms of a characteristic magnetic flux and a non dimensional variable $\psi=a R_{0} \bar{B}_{p}(a) \tilde{\psi}$, where 
$\bar{B}_{p}(a)=\mu_{0} I_{p} / 2 \pi a$ is the poloidal field on the surface of a cylindrical uniform current of radius $a$. Finally, the pressure may be written as $p(\psi)=p_{0} \tilde{p}(\tilde{\psi})$, with $p_{0}$ the maximum plasma pressure and $F(\psi)=R_{0} B_{0} \tilde{F}(\tilde{\psi})$, where $B_{0}$ is the vacuum magnetic field at the origin (of the local coordinates). The Grad-Shafranov, may then be written in non dimensional form as

$$
\tilde{\nabla}^{2} \tilde{\psi}-\frac{\epsilon}{1+\epsilon \tilde{r} \cos \theta}\left(\cos \theta \frac{\partial \tilde{\psi}}{\partial \tilde{r}}-\frac{\sin \theta}{\tilde{r}} \frac{\partial \tilde{\psi}}{\partial \theta}\right)=-\frac{1}{2}\left[\tilde{\beta}(1+\epsilon \tilde{r} \cos \theta)^{2} \frac{d \tilde{p}}{d \tilde{\psi}}+\frac{B_{0}^{2}}{\bar{B}_{p}^{2}} \frac{d \tilde{F}^{2}}{d \tilde{\psi}}\right]
$$

Where $\tilde{\beta}=2 \mu_{0} p_{0} / \bar{B}_{p}^{2}(a)[28]$, is defined in analogy to $\beta \equiv p /\left(p+B^{2} / 2 \mu_{0}\right)$, that measures the effectiveness of plasma confinement [2], and varies little inside the plasma. From here, the non dimensional form will be used, then we will exclude the $\sim$ in the calculations (except for $\tilde{\beta}$ ).

Until now, no approximations have taken place, eq.(3.58) is exact. Then we can perform a perturbative expansion of the poloidal flux in powers of the inverse aspect ratio $\epsilon$. This is strictly valid for large aspect ratio tokamaks where the toroidal effects are small. Modern tokamaks have a small aspect ratio, but the use of the perturbative expansion gives a good insight about the toroidal effects on the equilibrium. Clearly, when $\epsilon \rightarrow 0$ we recover the cylindrical limit, that is, essentially, an unidimensional problem, then we require the first term on the perturbarive expansion to depend only on $r$. We have

$$
\psi(r, \theta)=\psi_{0}(r)+\epsilon \psi_{1}(r, \theta)+O\left(\epsilon^{2}\right)
$$

Introducing this into eq.(3.58), expanding the operator in powers of $\epsilon$ and matching powers of the inverse aspect ratio, we obtain the zero and first order equations

$$
\begin{aligned}
& \nabla^{2} \psi_{0}=-J\left(\psi_{0}\right) \\
& \nabla^{2} \psi_{1}-\cos \theta \frac{d \psi_{0}}{d r}=-\frac{d J}{d \psi_{0}} \psi_{1}-\tilde{\beta} r \cos \theta \frac{d p}{d \psi_{0}}
\end{aligned}
$$

where the non dimensional current is

$$
J(\psi)=\frac{1}{2}\left(\tilde{\beta} \frac{d p}{d \psi}+\frac{B_{0}^{2}}{\bar{B}_{p}^{2}} \frac{d F^{2}}{d \psi}\right) .
$$

As the first equation is purely radial, we can write $\nabla^{2}=r^{-1} d / d r(r d / d r)$. The solution to (3.60) depends entirely on the choice of the source functions. Although this equation is, in general, nonlinear, the existence of solutions is always guaranteed for a reasonable choice of $J(\psi)$. The first order equation may be simplified by the introduction of the 
ansatz $\psi_{1}(r, \theta)=f(r) \cos \theta$ and after some manipulations [28], we obtain

$$
f(r)=\frac{d \psi_{0}}{d r}\left[C+\int_{0}^{r} d x \frac{1}{x\left(\frac{d \psi_{0}}{d x}\right)^{2}} \int_{0}^{x} d y\left[y\left(\frac{d \psi_{0}}{d r}\right)^{2}-\tilde{\beta} y^{2}\left(\frac{d p\left(\psi_{0}\right)}{d y}\right)\right]\right] .
$$

The value of $C$ is defined from the boundary conditions. For instance, we can ask for the last magnetic surface of the plasma to be the circle $r=a$. This means that the angular dependence must vanish for $r=a$ or $f(a)=0$. Now, if we define $g(r)=f(r) / r$ the first order solution may be written

$$
\psi(r, \theta)=\psi_{0}(r)+g(r) r \cos \theta=\psi_{0}(r)+g(r) x
$$

with $x=r \cos \theta$. Taking the gradient of this quantity we have

$$
\nabla \psi=\psi_{0}^{\prime} \hat{\boldsymbol{r}}+g^{\prime} x \hat{\boldsymbol{r}}+g \hat{\boldsymbol{x}}
$$

where "'" indicates radial derivative. Recalling that the poloidal field is $\boldsymbol{B}_{p}=\nabla \psi \times \nabla \phi$, the magnetic axis will appear when the field is purely toroidal or $\nabla \psi=0$. In terms of (3.65), the condition for the existence of magnetic axis reads

$$
\left(\psi_{0}^{\prime}+g^{\prime} x\right) \hat{\boldsymbol{r}}+g \hat{\boldsymbol{x}}=0
$$

This equation can be satisfied in two ways, the first is that the conditions $g\left(r_{c}\right)=0$ and $\psi_{0}^{\prime}\left(r_{c}\right)+g^{\prime}\left(r_{c}\right) x_{c}=0$ occur simultaneously. If $r_{c} \neq x_{c}$, this condition leads to symmetric up-down magnetic axes relative to the plane $z=0$. For simplicity lets hold up the analysis of this configuration to further sections.

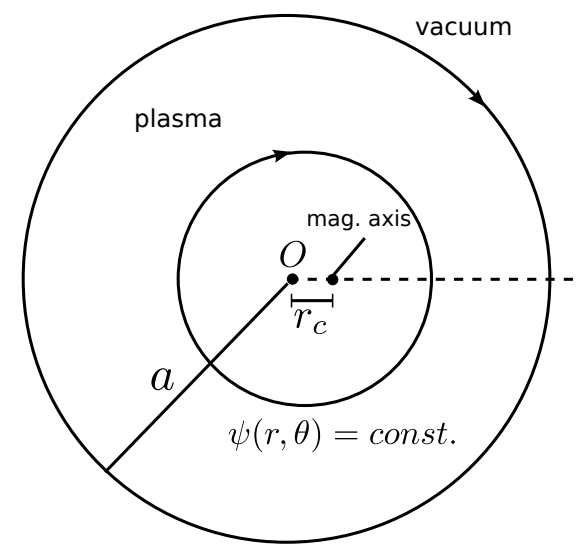

Figure 3.7: Shafranov shift, in the case of a single magnetic axis.

The second way to get a vanishing poloidal flux is that the unit vector $\hat{\boldsymbol{r}}$ points in the 
same direction that $\hat{\boldsymbol{x}}$. This is true for $\theta=0, \pi$, leading to the conditions

$$
\psi_{0}^{\prime}\left(r_{c}\right)+g^{\prime}\left(r_{c}\right) r_{c} \pm g\left(r_{c}\right)=0
$$

Clearly, the choice of the source functions defines the topology of the solutions. Those may lead to the existence of several magnetic axis and hyperbolic points in the plasma domain. For now, lets assume that the tokamak behavior does not differ much from that of a straight plasma current surrounded by a cylindrical conducting wall.

If we ask for (3.67) to be satisfied just once, say for $\theta=0$, then we have just one magnetic axis shifted from the origin (fig.3.7), this is called Shafranov shift, and is one of the experimentally recognizable toroidal effects. In general terms, the equilibrium problem is a two dimensional one, a realistic poloidal flux $\psi$ is not expected to have any remarkable symmetries about its magnetic axes, mostly today, that most of working tokamaks have $\epsilon>1 / 4$.

\subsubsection{Safety Factor}

The safety factor is an important function characterizing an equilibrium configuration. Its value is well defined over any closed magnetic surface, so it is a flux function. The function $q(\psi)$ is defined as the number of toroidal turns performed by a magnetic line in its magnetic surface as it performs a poloidal cycle. The value of $q(\psi)$ may be rational or irrational entailing the fact that the corresponding magnetic lines are closed or not. When $q(\psi)$ is irrational we name the corresponding torus an irrational surface and the magnetic lines fill it ergodically.

This function may be calculated by evaluating the integral

$$
q(\psi)=\frac{1}{2 \pi} \int_{0}^{2 \pi}\left[\frac{r B_{\phi}}{R B_{p}}\right]_{\psi} d \theta
$$

with $(r, \theta)$ the local polar coordinates about the magnetic axis, and $R$ the cylindrical coordinate. $B_{p}$ and $B_{\phi}$ are the poloidal and toroidal magnetic fields respectively and the integral is to be calculated on a fixed magnetic surface.

The safety factor have some relevant properties in terms of robustness of the magnetic surfaces against nonaxisymmetric perturbations. For ideal plasmas $q(\psi)$ is the analogue in to the canonical period of the orbits in hamiltonian systems, the helicity of a given invariant torus. In absence of reconnection the presence of nonaxisymmetric perturbations is expected to destroy the rational surfaces, creating resonant magnetic islands in a sea of chaos. Also, most of the irrational surfaces are expected to survive under a sufficiently weak perturbation. 


\section{Chapter 4}

\section{Current Reversal Equilibrium RESULTS}

Over the last few years, the experimental realization of non-monotonic current profiles in tokamaks has attracted ever increasing attention. A reduction in the toroidal current density, near the magnetic axis, leads to a non-monotonic safety factor $q(\psi)$, that, in the context of hamiltonian dynamics, is related to the existence of a shearless torus. For non-integrable systems this is important, for instance, when considering the localized asymmetries implicit to the tokamak design (like discreteness of the toroidal field coils, diagnostic elements, etc), periodic perturbations ${ }^{1}$ of the poloidal flux appear and destruction of rational magnetic surfaces occurs. For $q(\psi)$ monotonic, the destruction of magnetic surfaces follows the description given by the KAM theory [20], where the most irrational tori preserve their topology under perturbations. When the perturbation strength is increased, chaotic regions near two different resonances may fuse, leading to the wandering of magnetic lines over broad regions inside the tokamak; this means that charged particles may be transported to external regions by the field lines themselves, giving rise to a zero order lost of fusion material.

In the non-monotonic scenario, a very robust transport barrier appears and encloses the internal region of the tokamak $[9,29]$. Numerical studies, using discrete maps, have shown that this structure is persistent under a wide range of values of the control parameters [30]. Even after its destruction, the barrier region affects dramatically the evolution of orbits, that spend large times in one region without crossing the shearless barrier. Hollow current profiles may provide some basic mechanisms for particle confinement in tokamaks, even when magnetic surfaces have been destroyed. Understanding this "frozen time transport" may lead to better understanding of some enhanced confinement regimes.

The current reversal equilibrium is an hypothetical configuration, in which the toroidal

\footnotetext{
${ }^{1}$ Recall that the toroidal angle is related to the time in the hamiltonian formulation.
} 
current density may reach negative values at some regions of the tokamak; it appear as a natural extension of current hollow configurations, allowing the hollow to reach negative values. The existence of this negative hollow is conjectured from the fact that, for some experimental hollow configurations, the current density near the magnetic axis attains almost zero values, and the error in the measurement includes negative values in a finite region. In the present work, our aim is to show that current reversal equilibrium, if possible, must present a global change in the equilibrium topology.

\subsection{Comments on Stability}

As was pointed in the previous chapter, the G-S equation describes a wide variety of equilibrium configurations, but it does not provide information about their stability. Then, before starting with the fundamentals of equilibrium in current reversal scenarios, a little digression on stability is in order.

Assume we have a single loop of toroidal current. As this is an axisymmetric configuration, the poloidal flux $\psi$ can be used to describe the topology of the field lines. It can be shown [19], that for a single loop of radius $a$ at the plane $z=0$, the poloidal flux function becomes

$$
\psi_{a}(R, z)=\frac{\mu_{0} I}{2 \pi} \frac{\sqrt{a R}}{k}\left[\left(2-k^{2}\right) K(k)-2 E(k)\right],
$$

with $k^{2}=4 a R /\left[(a+R)^{2}+z^{2}\right]$ and $\{E(k), K(k)\}$, complete elliptic integrals. In the plane $\phi=$ const., the magnetic field lines encircles the current loop (fig.4.1), then any test toroidal current ${ }^{2}$ experiences, locally, a force orthogonal to the magnetic surfaces; in the whole ring, the net force is along the symmetry axis. As expected, a test current of the same sign that the source of the magnetic field will tend to approach to it, and an opposite test current will tend to move away from the field source.

Considering two current loops, from the action-reaction law, the rings will suffer equal but opposite forces (fig. 4.2). In the case of opposite currents the system only finds equilibrium if both current loops are in the same plane, but an arbitrarily small displacement introduces a destabilizing force that separates both rings along the symmetry axis; also an arbitrarily small rotation about any line in the plane $z=0$, introduces a stabilizing torque that tends to line up the currents in a stable configuration.

The presence of this effects may lead us to think that current reversal equilibrium configurations may be highly unstable against the unavoidable perturbations in any experimental setup. Nevertheless, this assumptions comes from the discrete nature of the considered currents; in the previous scheme, a well localized current produces a well

\footnotetext{
${ }^{2}$ This means that the magnetic field created by the test current is not considered, since it does not affect its own equilibrium (clearly we are talking about a rigid ring).
} 


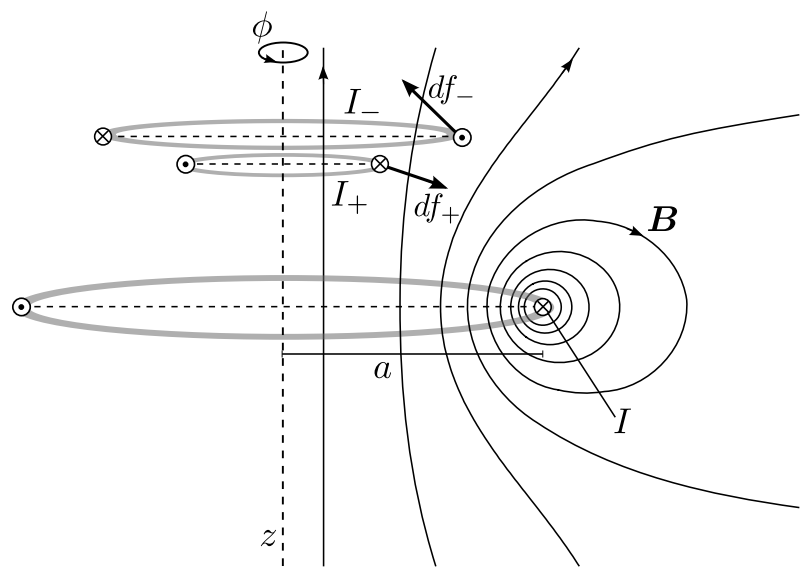

Figure 4.1: Magnetic field due to a finite radius current loop.

defined field in the points occupied by another well known current distribution. In equilibrium, a plasma is a self-consistent structure, the plasma currents distributes over a finite domain and the magnetic and kinetic forces balance at each point in the space; we can not consider that the magnetic field produced by a given region of the plasma directly affects the currents in other region, since the plasma is not a linear medium, and the field produced is subjected to local variations due to the plasma current. We can only make safe comments about stability of an equilibrium configuration by performing a first order expansion of the one-fluid equations (3.21- refdivergenceless), about a previously calculated current reversal equilibrium configuration; however, in this work we will be concerned with the equilibrium and its topology, so a stability analysis goes beyond the scope of the present work.
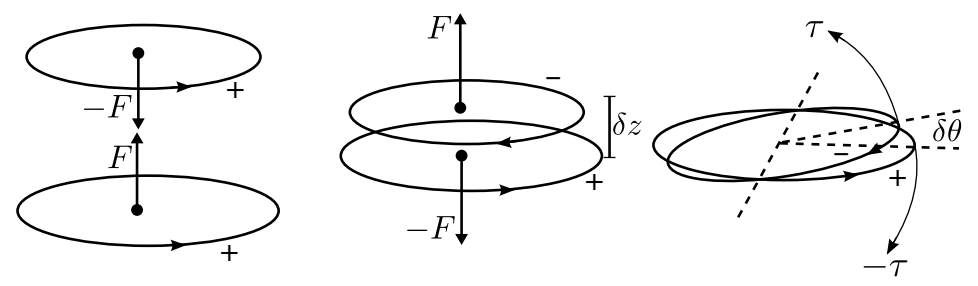

Figure 4.2: Stabilizing and destabilizing effects

\subsection{Topology of the CRECs}

Assuming equilibrium and axisymmetry, we are in the integrable case, meaning that we have well defined magnetic surfaces, and we can label them with the poloidal flux value ${ }^{3}$ $\psi$. The magnetic lines remains attached to such surfaces, that are also isobars of the

\footnotetext{
${ }^{3}$ Such labeling is only possible for families of nested magnetic surfaces, so that it is not unique when more that one magnetic axis exists.
} 
plasma. With this basic properties common to all equilibrium solutions it is possible to perform a geometrical study of the magnetic surfaces in presence of a negative current density. To do this, lets start with the Ampere's law in its integral form.

$$
\nabla \times \boldsymbol{B}=\mu_{0} \boldsymbol{j} \Rightarrow \oint_{\gamma} \boldsymbol{B} \cdot d \boldsymbol{l}=\mu_{0} I_{t}
$$

where the circuit $\gamma$ encloses a region in some plane $\phi=$ const., through which a net toroidal current $I_{t}$ flows. Now, if the toroidal current density may become negative, we can build several circuits enclosing regions through which the net toroidal current vanishes (fig. 4.3).

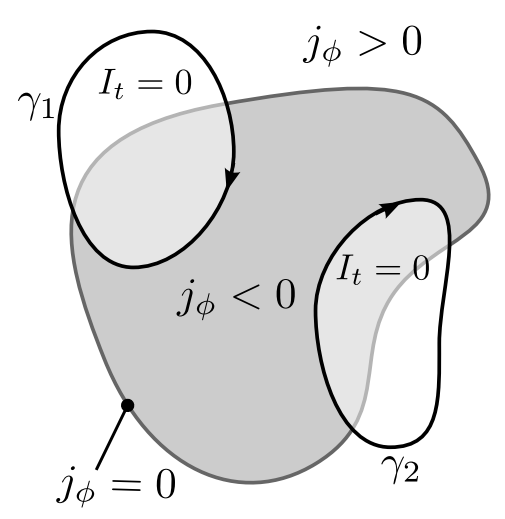

Figure 4.3: Circuits containing zero plasma current.

We expect the solution to the Grad-Shafranov equation to be a smooth axisymmetric function, then its level surfaces defines families of smooth curves in any plane $\phi=$ const. This set of curves may be labeled with the magnetic flux, so we will name them "magnetic circuits". For a magnetic circuit the Ampere's law is written

$$
\oint_{\psi} B_{p} d l=\mu_{0} I_{t}(\psi)
$$

where clearly $I_{t}$ is, by construction, a function of $\psi$. Now, if reversed current densities are possible in equilibrium, there is a closed circuit enclosing a vanishing toroidal current. By consistency of the magnetic surfaces orientation, the poloidal field attached to a given magnetic surface can not reverse its direction, then $\oint B_{p} d l$ can only vanish if the poloidal field is zero everywhere on the circuit; this implies that the total magnetic field is toroidal on the whole magnetic surface. Lets assume for a while that such circuit exists; in previous sections has been shown that the poloidal field may be written as

$$
\boldsymbol{B} p=\nabla \psi \times \nabla \phi=\frac{\nabla \psi \times \boldsymbol{\phi}}{R} .
$$


Then, a vanishing poloidal field implies directly $\nabla \psi=0$, given that $\nabla \psi$ does not have a toroidal component. Then, the zero current circuit is defined over a magnetic surface with zero gradient everywhere. Before going on, recall that the equilibrium problem is intrinsically two-dimensional, and the poloidal flux function is in general a two-variables function $\psi(R, z)$. If we ask for a vanishing $\nabla \psi$, this means, in cylindrical coordinates

$$
\frac{\partial \psi}{\partial R}=0, \frac{\partial \psi}{\partial z}=0
$$

The first equation establishes a relation between $R$ and $z$, defining one or several curves where the condition $\partial_{R} \psi=0$ is satisfied, similarly, the second equation leads to another set of curves. The intersections of this two families of curves defines the points where $\nabla \psi=0$. In general, two different families of curves intersect at a number of isolated points; continuous intersections are a signature of a highly degenerated situation induced by the two-dimensional representation of a intrinsically unidimensional problem. An infinite degeneracy is, of course, possible for scalar fields with special symmetries; but, asking for $\psi=$ const and $\nabla \psi=0$ over the same curve is just too restrictive, for any general scalar field without very remarkable symmetries. If we expect the reversed current configurations to be robust, they must be possible in non-symmetric configurations of the magnetic flux.

After this, the most general way to obtain a vanishing plasma current is to find a magnetic surface where the poloidal field reverses its direction. As was told before, this can't happen for a regular magnetic circuit, unless that the magnetic surface is not uniquely defined at a given toroidal curve. Lets assume for a while that this actually happens.

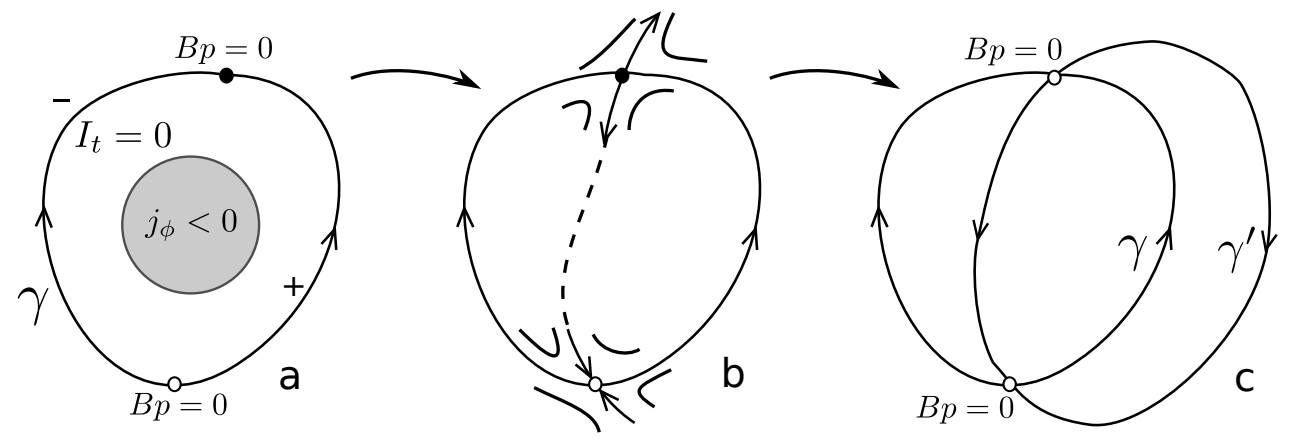

Figure 4.4: Creation of magnetic islands due to the reversed current.

Assume, by consistency, that we have a magnetic circuit with two critical points where the poloidal field vanishes (Figure 4.4a). As the magnetic field is conservative, those critical points can not be sources or sinks, and of course are not elliptic, since the magnetic circuit goes through them; then we have two hyperbolic points, implying the existence intersecting magnetic surfaces that correspondingly meet at the zero poloidal field points 
(Figure 4.4b). Now, as we are in the integrable case those new magnetic surfaces must connect the zero points by inside and probably outside ${ }^{4}$ the initially considered magnetic circuit. In conclusion, we have formation of magnetic island structures due to the existence of a reversed current density (Figure 4.4c). Finally, as we expect the poloidal flux $\psi(R, z)$, to be a continuous function, the pair of curves $\left\{\gamma, \gamma^{\prime}\right\}$ linking the hyperbolic points must be labeled by the same poloidal flux value, even more, each of the intersecting circuits are expected to be smooth.

Recall that the initial circuit $\gamma$ encloses a zero toroidal current, then the total toroidal current passing through the whole islands structure is provided by the region inside $\gamma^{\prime}$ and outside $\gamma$. From the orientation of the poloidal field respect to the curve enclosing this region, is clear that the toroidal current of the whole system is positive.
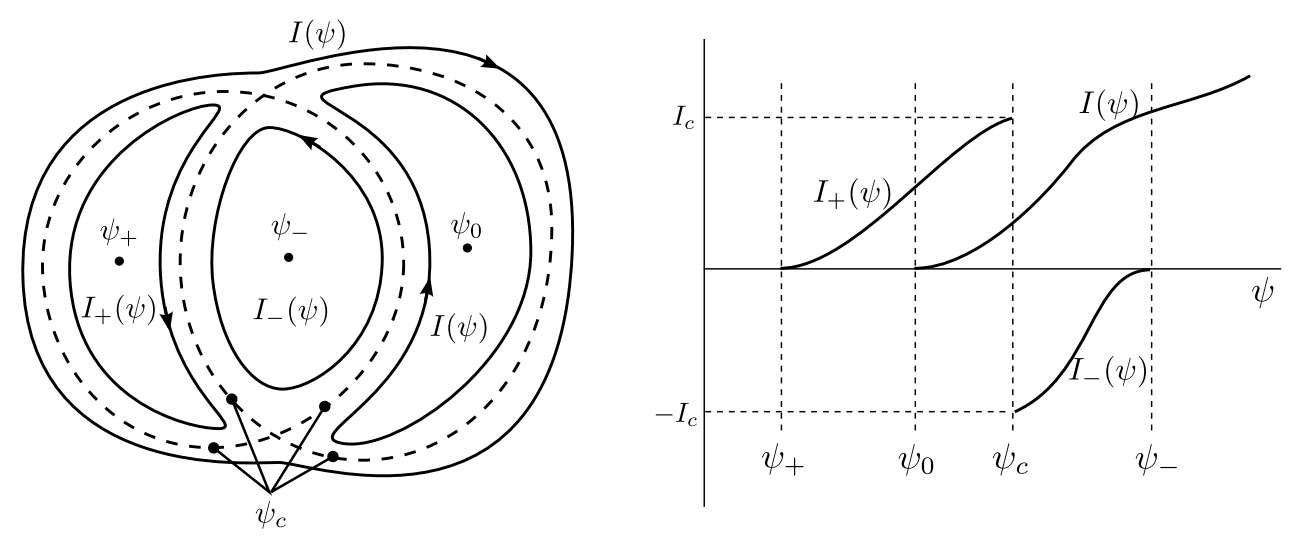

Figure 4.5: The toroidal current as a function of the poloidal flux, for different families of nested surfaces.

In fig.4.5, we can see the different domains where we can define the functions $I_{t}(\psi)$, about each of the three magnetic axes. Each current start in zero, and changes when moving out in each family of nested islands. At the surface $\psi_{c}$ two of the currents $\left(I_{+}, I_{-}\right)$ must reach opposite values. The remaining current $(I)$, is continuous over any path, going from $\psi_{0}$, to the surfaces outside the system, without passing through the other families of nested surfaces.

\subsubsection{Generalization}

Now that we have introduced the basic mechanisms for magnetic island formation in reversed current scenarios we can generalize the idea and consider different geometries that may contain current reversals. The most simple scenarios come from the intersection of two simple circuits; a principal one, enclosing a vanishing plasma current and a secondary one intersecting the first, an even number of times. This leads to an even system of

\footnotetext{
${ }^{4}$ This is true whenever there are not other hyperbolic points in the system.
} 
magnetic islands about a central structure with negative plasma current (Figure 4.6). Note that the resulting current of the full structure is always positive, since the poloidal field just outside the island chain winds oppositely to that in the central region. This is due to the contribution to the current inside the secondary magnetic circuit and outside the primary one.
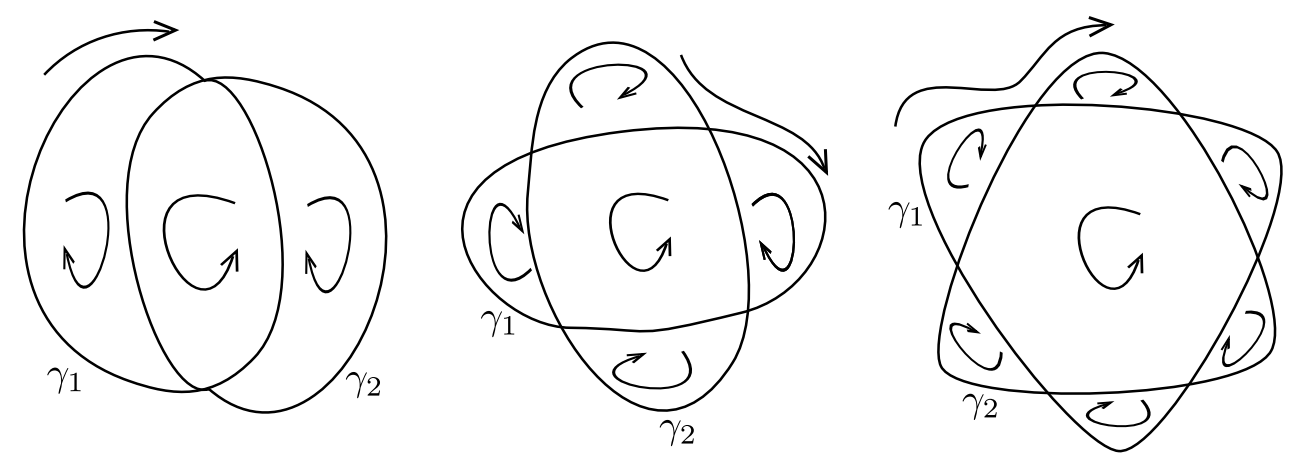

Figure 4.6: Even systems of islands, from the intersections of two simple magnetic circuits.

In fig.4.6, we can see the three first systems of islands that may be created from the intersections of two simple smooth curves. The arrows shows the overall behavior of the poloidal field for each domain of the plane. Outside the islands system, the poloidal field encloses the whole structure and further magnetic surfaces in outer regions, show less the shape influence of the nonested magnetic surfaces.

\subsubsection{Non-simple circuits}

Slightly more complicated geometries may appear if we allow a single circuit to selfintersect. In this case the circuit remains differentiable, but does not have a well defined internal region. The most simple case is shown in fig. 4.7, where a self-intersecting circuit "encloses" a zero plasma current; but to perform any calculation we must split the circuit in two simple (non-differentiable) ones.

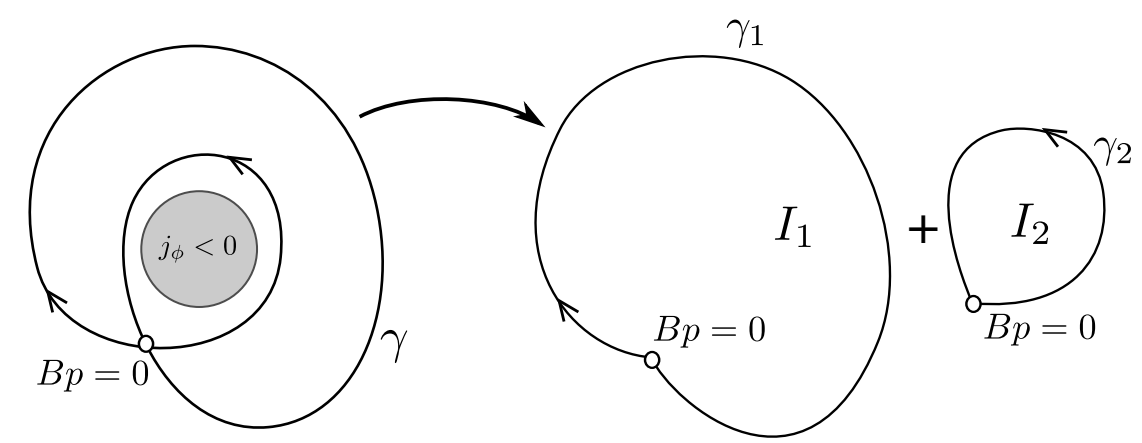

Figure 4.7: The most simple self-intersecting geometry. 
Integration of $B_{p}$ over $\gamma_{1}$ gives the total current enclosed by the structure; but as $\gamma=\gamma_{1}+\gamma_{2}$, then the current "enclosed" by the circuit $\gamma$ satisfies $I=I_{1}+I_{2}$. As $\gamma$ was chosen so that $I=0$ we get $I_{2}=-I_{1}$, then the total current enclosed by the structure is the negative of the current enclosed by the internal structure, in other words, a positive current. This result is similar to that obtained in the intersections of two differentiable circuits.

In Figure 4.8 we can see a generalization of this structure to include more magnetic islands produced by a self-intersecting circuit. Obviously, a single smooth curve can only self-intersect an odd number of times, to form odd island systems. This complements the structures of fig. 4.6. Again the total current through the whole structure is the negative of the internal one, i.e. is positive.
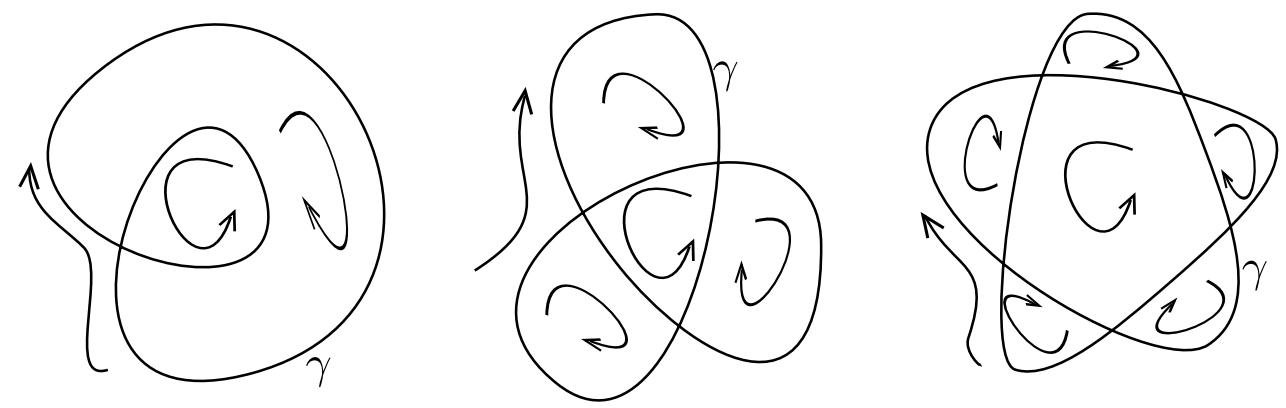

Figure 4.8: Odd systems of islands from a self-intersecting differentiable magnetic circuit.

Noticeably, the assumption of a current reversal inside an axisymmetric plasma, gives naturally the obtained topologies. Only the Maxwell equations and the existence of a smooth poloidal flux function are required. It is expected then, that the Grad-Shafranov equation leads naturally to this topologies when negative hollow current profiles are considered; this will be explored in the next chapter. An important feature of this equilibria, may be the chaotic behavior of the magnetic field near the critic surfaces, since hyperbolic points are most sensitive to the introduction of periodic perturbations, due to homoclinic or heteroclinic chaos. Under the influence of external perturbations, the magnetic field lines are allowed to wander between the internal and external regions of the chain of magnetic islands.

In recent works [31-33], after the choice of particular source functions, the reduction of the G-S equation to the linear case makes available analytical forms of the poloidal flux function (or the toroidal one in [31]). In the 2003 Martynov et. al. [32], linearization is used as a starting point for driving a numerical approach to the equilibrium by means of the CAXE code [34]. In each case, after the introduction of a current reversal configuration, the magnetic islands appears, always under the scheme of two intersecting or one selfintersecting critic magnetic circuit (fig. 4.9). However, the restrictions of this methods 

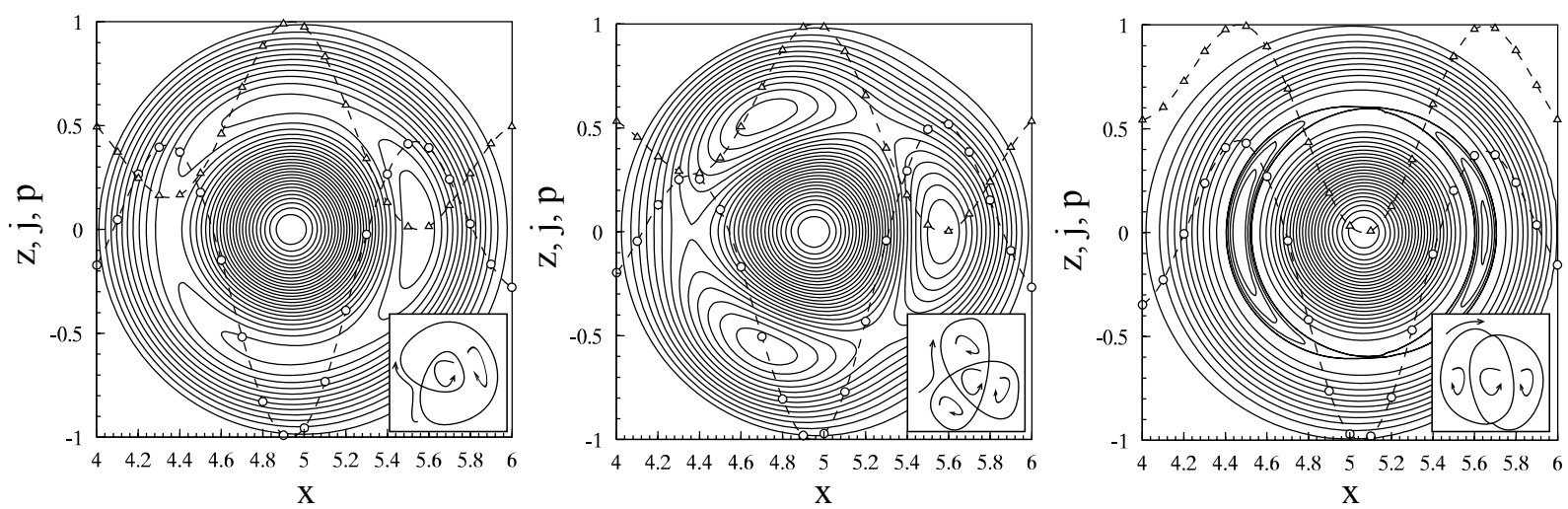

Figure 4.9: Equilibrium configurations for a particular choice of the source functions (from [31]), the normalized current density (open circles) exhibit a negative hollow and the normalized pressure (open triangles) is maximum at the magnetic axis. In the small boxes we show the corresponding structures of critic curves leading to each topology.

comes from the particular choices of the source functions, that sometimes makes the current hollow too wide and the negative value of the current, too large, which is an overestimation of the expected effects near a positive hollow configuration; also, strong assumptions about the global structure of the plasma and its edge are introduced. In the following, our aim is to show that the magnetic islands may be addressed in a local way, and there is no need to assume a particular form of the source functions nor establishing boundary conditions at the plasma edge.

\subsection{Local Solution to the Grad-Shafranov Equation}

In the following, we will develop an scheme formally similar to the successive approximations method, just that in our case we are not interested in describing the global behavior of the flux function, but just a small region of the plasma.

We start by assuming that there is a tiny region in the tokamak where the current density is negative. This implies that there is a closed curve in the poloidal plane where the current density vanishes (fig. 4.10). As we know, the toroidal current density may be written

$$
j_{\phi}=R \frac{d p}{d \psi}+\frac{F}{\mu_{0} R} \frac{d F}{d \psi}
$$

where $p(\psi)$ and $F(\psi)$ are arbitrary functions depending on the particular equilibrium. The condition $j_{\phi}=0$ becomes $\mu_{0} R^{2} p^{\prime}+F F^{\prime}=0$, where was used that in the plasma region $R \neq 0$. As we expect the negative hollow to be small, $R$ must change very little in the previous expression. This means that the condition $j_{\phi}=0$ is approximately satisfied in a flux surface. 


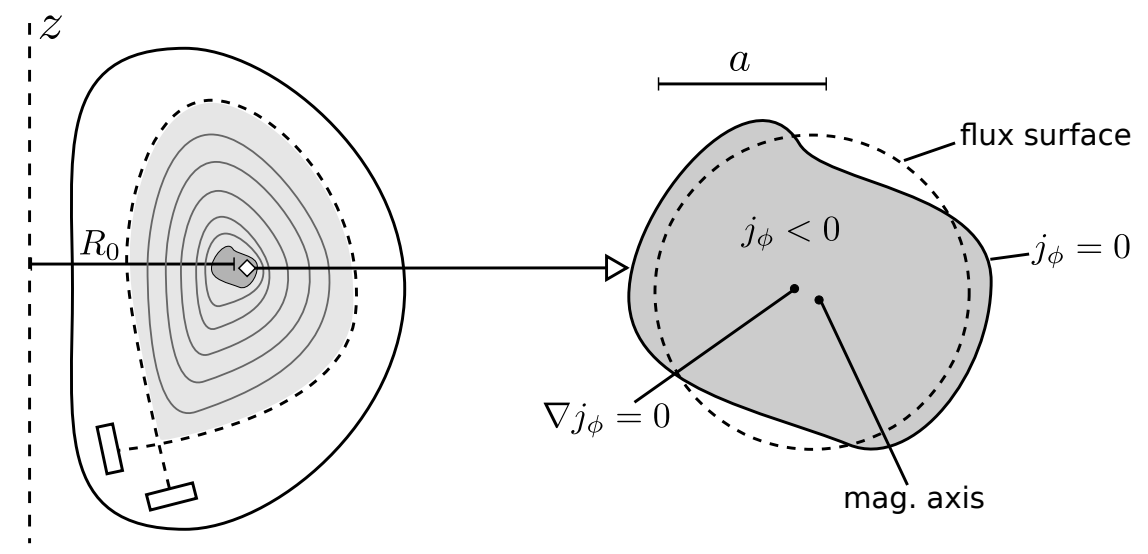

Figure 4.10: In a small region inside the tokamak, the large aspect-ratio approximation is very accurate.

Somewhere inside the region, there is a critical point of the toroidal current density, a point where $\nabla j_{\phi}=0$. Also, near the curve $j_{\phi}=0$, there is a closed magnetic surface, so that in the region is also a magnetic axis. This axis, in general, is not at the same position that the critical point of the current density; but those are expected to be near, so we will perform our expansions about the critical point in the toroidal current density. We can introduce a characteristic distance $a$, within which this local description is expected to be valid (i.e. the expansions about the magnetic axis are good), then we can nondimensionalize the problem. Notice that this local description can be made for any configuration under the assumption of current reversal and here the inverse aspect ratio $\epsilon$ is a truly small parameter (fig.4.10).

The G-S equation may be written as

$$
\Delta^{*} \psi=-\mu_{0} R j_{\phi}
$$

Since we are near a minimum in the current density, we can perform a Taylor expansion about this critical point, where the linear term (in the position) vanishes. To quadratic terms in $\boldsymbol{r}$ we have:

$$
j_{\phi}\left(\boldsymbol{r}_{0}+\delta \boldsymbol{r}\right)=j_{\phi}\left(\boldsymbol{r}_{0}\right)+(\delta \boldsymbol{r} \cdot \nabla) j_{\phi}+\frac{1}{2}(\delta \boldsymbol{r} \cdot \nabla)^{2} j_{\phi}=j_{0}+\delta \boldsymbol{r}^{T} H\left(j_{\phi}\right) \delta \boldsymbol{r} .
$$

A further simplification suggests the current to be parabolic, then the Hessian matrix $H\left(j_{\phi}\right)$ is proportional to the identity. In the more general case, the local description of the current density is an elliptic paraboloid, with its symmetry axes rotated. To introduce the basic mechanisms leading to magnetic island formation, this accuracy is not necessary, but when considered, some more topological devises are available. However, for the time being, in local polar coordinates we can write $j_{\phi}(r)=j_{0}+\iota r^{2}$. Using $R=R_{0}+r \cos \theta$ 
and introducing nondimensional variables, $r=a \tilde{r}, \psi=\psi_{c} \tilde{\psi}$, we have

$$
\tilde{\Delta}^{*} \tilde{\psi}=\alpha(1+\epsilon \tilde{r} \cos \theta)\left(1+\eta \tilde{r}^{2}\right)
$$

where we defined $\alpha=-\mu_{0} j_{0} R_{0} a^{2} / \psi_{c}, \eta=\iota a^{2} / j_{0}$ and $\tilde{\Delta}^{*}$ is like in (3.57). Clearly, this is a local description, since the current density can not keep growing to the plasma edge. Removing all the tildes, we are left with the following dimensionless problem

$$
\nabla^{2} \psi-\frac{\epsilon}{1+\epsilon r \cos \theta}\left(\cos \theta \frac{\partial \psi}{\partial r}-\frac{\sin \theta}{r} \frac{\partial \psi}{\partial \theta}\right)=\alpha(1+\epsilon r \cos \theta)\left(1+\eta r^{2}\right)
$$

with $\nabla^{2}$ the Laplace operator in a plane. Asking for $\epsilon$ to be small, we write the perturbative expansion

$$
\psi(r, \theta)=\psi_{0}+\epsilon \psi_{1}(r, \theta)+O\left(\epsilon^{2}\right) .
$$

To zero order we have

$$
\frac{1}{r} \frac{d}{d r}\left(r \frac{d \psi_{0}}{d r}\right)=\alpha\left(1+\eta r^{2}\right)
$$

integrating between 0 and $r$

$$
\frac{d \psi_{0}}{d r}=\frac{\alpha}{4}\left(2+\eta r^{2}\right) r
$$

and integrating again, asking for $\psi_{0}(0)=0$ we obtain

$$
\psi_{0}(r)=\frac{\alpha}{4}\left(1+\frac{\eta}{4} r^{2}\right) r^{2}
$$

To first order we have

$$
\nabla^{2} \psi_{1}-\cos \theta \frac{d \psi_{0}}{d r}=\alpha r \cos \theta\left(1+\eta r^{2}\right)
$$

using (4.13), and defining $x=r \cos \theta$, the equation becomes

$$
\nabla^{2} \psi_{1}=\alpha x\left(\frac{3}{2}+\frac{5}{4} \eta r^{2}\right)
$$

Now, by simplicity, let us assume that $\psi_{1}$ can be separated as $\psi_{1}(r, \theta)=x f(r)$; otherwise we have to solve a non-separable elliptic ODE. This ansatz leads to $\nabla^{2} \psi_{1}=x\left(\nabla^{2} f+\right.$ $\left.2 f^{\prime} / r\right)$, where "'", indicates $r$-derivative. With this, and the radial part of the $\nabla^{2}$ operator we get

$$
\frac{d^{2} f}{d r^{2}}+\frac{3}{r} \frac{d f}{d r}=\alpha\left(\frac{3}{2}+\frac{5}{4} \eta r^{2}\right)
$$


multiplying by $r^{3}$ and reordering

$$
\frac{d}{d r}\left(r^{3} \frac{d f}{d r}\right)=\alpha r^{3}\left(\frac{3}{2}+\frac{5}{4} \eta r^{2}\right)
$$

This can be integrated straightforwardly to obtain

$$
\psi_{1}(r, \theta)=\frac{\alpha}{16}\left(3+\frac{5}{6} \eta r^{2}\right) r^{3} \cos \theta .
$$

Then we can write the full solution, to the first order in $\epsilon$ as

$$
\psi(r, \theta)=\frac{\alpha}{16} \Phi(r, \theta)
$$

Here, the function $\Phi(r, \theta)$, contains the geometrical aspects of the flux function and may be written

$$
\Phi(r, \theta)=\left(4+\eta r^{2}\right) r^{2}+\epsilon\left(3+\frac{5}{6} \eta r^{2}\right) r^{2} x,
$$

with $x=r \cos \theta$. This is the desired analytical form of the poloidal flux function inside the small region of interest containing a critical point of the current density. With this, we can investigate the topology of the flux surfaces by finding the critical points of $\Phi$ where $\nabla \Phi=0$ is satisfied.

As the dependence on $\theta$ is only through the function $\cos \theta$, we may think that the poloidal flux is a function of $r$ and $x$, then we can write the gradient of $\Phi$ like

$$
\nabla \Phi=\frac{\partial \Phi}{\partial r} \nabla r+\frac{\partial \Phi}{\partial x} \nabla x
$$

In general $(\theta \neq 0, \pi)$, the vectors $\nabla r=\hat{\boldsymbol{r}}$ and $\nabla x=\hat{\boldsymbol{x}}$ are independent, then the condition $\nabla \Phi=0$ is satisfied when $\partial_{r} \Phi=0$ and $\partial_{x} \Phi=0$ simultaneously. For $\partial_{x} \Phi=0$ we require $r_{1}=0$ or $r_{2}=\sqrt{-18 / 5 \eta}$, that is only possible for $\eta<0$. The first condition corresponds to the elliptic point at the magnetic axis. Inserting $r_{2}$ into the condition $\partial_{r} \Phi=0$ leads to $x_{2}=-16 / 15 \epsilon$; however, this is only meaningful if $\left|x_{2}\right| \leq r_{2}$, that leads to the condition $|\eta|<3.164 \epsilon^{2}$ for the simultaneous vanishing of the $x$ and $r$ parts of the gradient. This condition defines a bifurcation in which two critical points, mirrored through the $x$ axis, collide with a third one (to be found) in $y=0$ as $\eta$ is increased in magnitude but kept negative. Notice that those critical points are at a distance of order $1 / \epsilon$ from the magnetic axis, then they are outside the reliable region, and the plasma will not necessarily exhibit them.

Now, we must consider the cases in which $\nabla r$ and $\nabla x$ are dependent; this occurs for $\theta=0, \pi$; where $x= \pm r$. This critical points are horizontally aligned with the magnetic 
axis at $r=0$. To obtain them we can replace $x= \pm r$ in $\partial_{r} \Phi+\partial_{x} \Phi=0$, that after some manipulations leads to

$$
\frac{2+\eta r^{2}}{\frac{9}{4}+\frac{25}{24} \eta r^{2}} \pm \epsilon r=0
$$

where the \pm indicates either $\theta=0$ or $\pi$ for the direction of the critical point. We can understand this result by studying the intersections of the function $g(r)=\left(2+\eta r^{2}\right) /\left(\frac{9}{4}+\right.$ $\frac{25}{24} \eta r^{2}$ ) with the lines $y= \pm \epsilon r$ (fig.4.11).

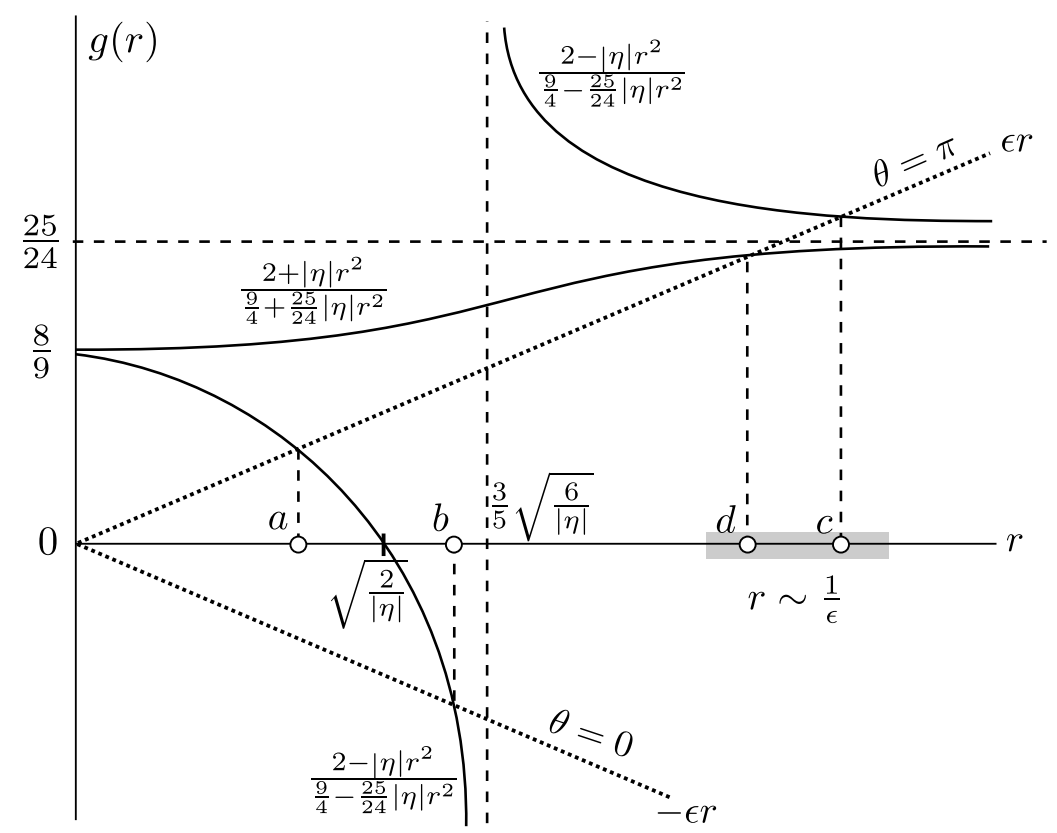

Figure 4.11: Illustration of the function $g(r)$ (continuous curves) for $\eta>0$ (sigmoid) and $\eta<0$ (asymptotic to $r=3 \sqrt{6} / 5 \sqrt{|\eta|}$ ) and the lines $\pm \epsilon r$ (dotted curves). The intersections between continuous and dotted curves, represents fulfillment of condition $(4.23)$

When $\eta>0, g(r)$ is like a sigmoid starting at $8 / 9$ and growing monotonically to $24 / 25$, as depicted in fig. 4.11; then it just intersects the line $\theta=\pi$ and such intersection occurs at a position of order $1 / \epsilon$ ( $d$ in the figure). This point is outside the region of interest, then in the area of interest the flux function exhibits a single magnetic axis at $r=0$ with the usual Shafranov shift of the magnetic surfaces. This is expected, since $\eta>0$ indicates a parabolic current profile without current reversal. When $\eta<0, g(r)$ is no longer monotonic, diverging at $r=3 / 5(\sqrt{6 /|\eta|})$ to $-\infty$ from the left and $\infty$ from the right (fig. 4.11). In this case $g(r)$ intersects the lines $y= \pm \epsilon r$ three times. For $\theta=\pi$ we have two intersections; one at a radius of order $1 / \epsilon$ like in the previous case $(c$ in fig. 4.11), and the other before the divergence of $g(r)$ ( $a$ in fig. 4.11). For $\theta=0$, we have another intersection before the divergence ( $b$ in fig. 4.11); then for a current reversal equilibrium $(\eta<0)$, two new critical points appears about the magnetic axis, at distances 
of the order $\sqrt{2 /|\eta|}$, so that $|\eta| \geq 2$ ensures the existence of such structures in a region where the description is valid.

From the Hessian matrix of $\Phi(r, \theta)$, it is also possible to show that $b$ is an elliptic point, and $a$ an hyperbolic one. The point $c$, changes from elliptic to hyperbolic after its collision with the two mirrored hyperbolic ones when $\eta$ exceeds $3.164 \epsilon^{2}$. In absence of other structures, the separatrixes merging at the hyperbolic point $a$, enclose the elliptic points (i.e. the magnetic axis $r=0$ and $b$ ), leading to a smooth self-intersecting magnetic circuit as predicted in the previous section.

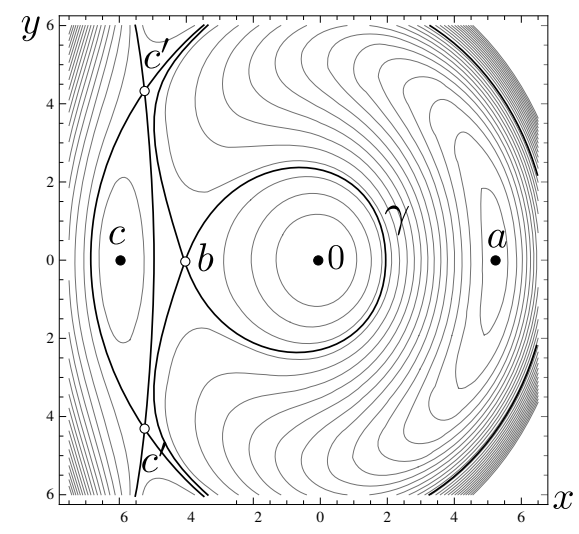

Figure 4.12: Topology for $\epsilon=0.2, \eta=-0.075936$, before merging of the $O(1 / \epsilon)$ critical points, exhibiting a self-intersecting magnetic circuit $\gamma$, about two magnetic axis $r=0$ and $a$.

In fig. 4.12 the control parameters were chosen so that the system exhibits most of its topology in a "large" region. The existence of two mirrored hyperbolic points off the plane $y=0$ is guaranteed since $|\eta|<3.164 \epsilon^{2}$; however this small value of $\eta$ moves the critical points $\{a, b\}$ outside the range of validity $(r \lesssim 1)$ of the model.

Further increase in the magnitude of $\eta$, (keeping it negative) leads to the collision of $c$ and the mirrored $c^{\prime}$ and moves $a$ and $b$ near the origin. As $\epsilon$ is reduced the inclination of the lines $\epsilon r$ and $-\epsilon r$ (fig. 4.11) becomes smaller, approaching the critical points $a$ and $b$ in the radial direction, reducing the size of the magnetic island about $a$.

In a more realistic situation (fig. 4.13), the hyperbolic point $c$ is far away from the critical points $a$ and $b$; and for those to be inside the reliable region, we require $\eta \gtrsim 2$. Recalling that $\eta=\iota a^{2} / j_{0}$, we see that reducing the size of the current density at the magnetic axis, leads to magnetic island formation inside the accuracy region. This is interesting, since experimental evidence only suggests the existence of a small negative current if any, due to the error bars of the current density at the zero density region in a hollow configuration. 

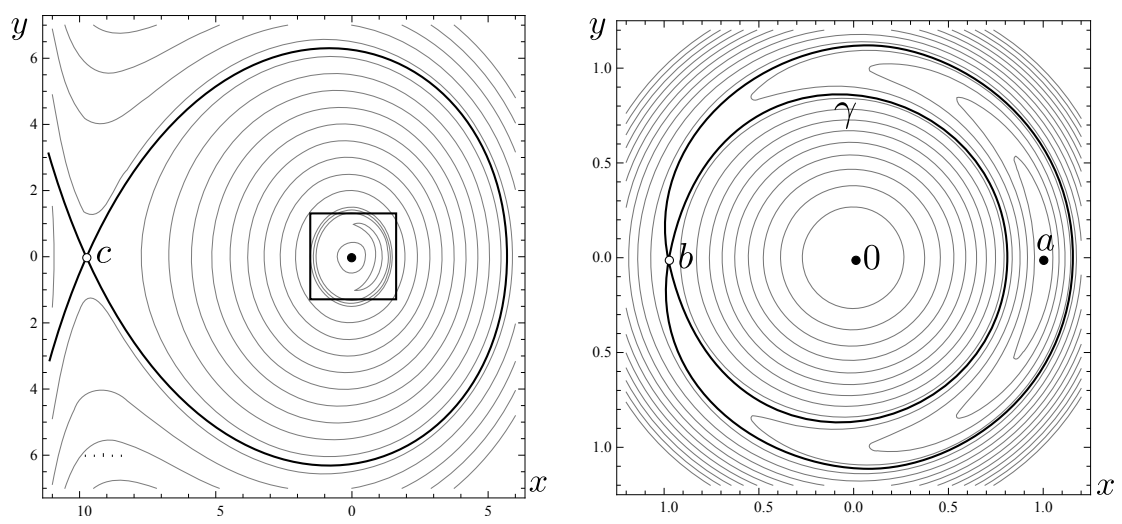

Figure 4.13: Global topology for $\epsilon=0.1, \eta=-2$ and zoom to the region of interest, after merging of the critical points at $r \sim O(1 / \epsilon)$. The system continues to present a self-intersecting magnetic circuit $\gamma$.

\subsubsection{Magnetic island width}

With the devised mechanisms, it is possible to estimate the width of the magnetic island. Lets start by noting that the function $\Phi(r, \theta)$ (eq. (4.21)) may be written

$$
\Phi(r, \theta)=f(r)+g(r) \cos \theta
$$

this function has a critical point at $a$ (fig. 4.13), then we can expand it like

$$
\Phi\left(r_{a}+\delta r, 0\right)=\Phi_{a}+\frac{1}{2} \Phi_{a}^{\prime \prime} \delta r^{2}
$$

in the horizontal direction. Given that the island is limited by the separatrixes coming from the hyperbolic point $b$, we must find $\delta r$ such that $\Phi\left(r_{a}+\delta r, 0\right)=\Phi\left(r_{b}, \pi\right)=\Phi_{b}$, then we can write $(4.25)$ as

$$
\Phi_{b}=\Phi_{a}+\frac{1}{2} \Phi_{a}^{\prime \prime} \delta r^{2}
$$

By performing a linear approximation about $r_{0}=\sqrt{2 /|\eta|}$, it is possible to show that $r_{b}-r_{a} \sim \epsilon / 6 \eta$ (fig. 4.11), then in practice we can use $r_{a} \sim r_{b} \sim r_{0}$; and using (4.24) for the points $a$ and $b$ we have

$$
\delta r^{2}=-\frac{4 g\left(r_{0}\right)}{\Phi_{0}^{\prime \prime}}
$$

then from (4.21) is easy to get $\Phi_{0}^{\prime \prime}=-\left(16+\epsilon 46 r_{0} / 3\right)$ and $g\left(r_{0}\right)=\epsilon 4 r_{0}^{3} / 3$. Inserting those in the previous equation we have

$$
\delta r^{2}=\frac{\epsilon}{3} \frac{r_{0}^{3}}{1+\frac{46}{48} \epsilon r_{0}},
$$


but we expect $r_{0} \lesssim 1$, and $\epsilon \ll 1$. Also, the total width $\Delta$ will be twice $\delta r$ and the island width is estimated about

$$
\Delta^{2} \sim \frac{4}{3} \epsilon r_{0}^{3}
$$

Then $\Delta$ goes like $\sqrt{\epsilon / \eta^{3 / 2}}$, as expected the island width reduces by increasing $\eta$, that means to increase the grow rate of the current density, or reduce the size of the minimum current density; then the nonested configuration effects would become more apparent for a wider current hollow. This result gives a good estimate in our configurations. Notice that (4.29) is written in terms of $r_{0}$, that can be easily measured for any CREC. It nearly coincides with the distance between the principal and secondary magnetic axes. Also $\epsilon$ may be defined from the region of interest where the previous approximations are considered good.

As an example, we take several one-island CRECs, and measure the relevant quantities in the arbitrary units provided in each publication (fig. 4.14). To use (4.29) we must measure all the lengths in terms of the size of the region of interest $a$. The value of $a$ may be fixed from the current profile, so that it covers a region where the current may be approximated by a paraboloid as in our model. However this condition can be relaxed, since our model is appropriate when the magnetic island is formed inside the region of interest, then a may be chosen more or less arbitrarily; with the condition that a circle with radius $r=a$ from the magnetic axis, contains the magnetic islands as a "relevant" structure. This appears to weaken our result, but several testings with different values of $a$ have shown that the resulting value of $\Delta$ does not strongly depends on this choice. In (fig 4.14), the size of $a$ has been taken, deliberately, as one-half the width of the plots frame in their respective units. With the chosen $a$ we can nondimensionalize the other quantities in each case, and calculate $\epsilon$ as well. Then we can insert the nondimensional critic radius $r_{0}$ and local aspect ratio $\epsilon$ into (4.29) and get the nondimensional $\Delta$. Finally, each value of $\Delta$ is multiplied by the corresponding $a$, to return to the arbitrary units.
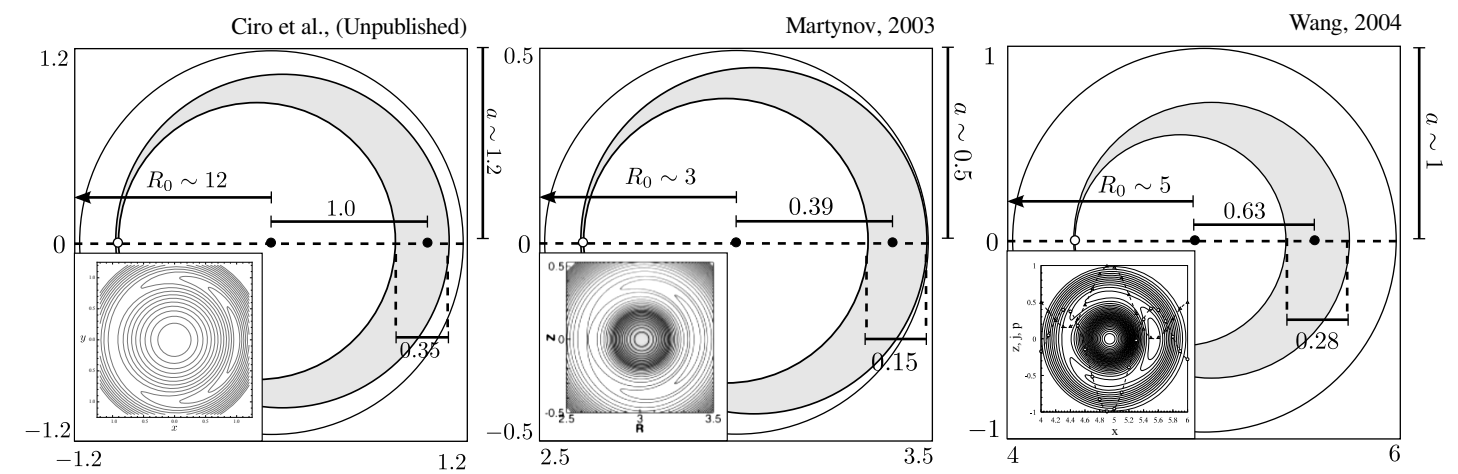

Figure 4.14: Relevant sizes of the one island structure in three different equilibria (from this work, [31] and [32], respectively.) 
From left to right in fig.4.14, island widths of $a \Delta=0.33,0.16$ and 0.26 (arbitrary units), were obtained with corresponding errors of $5.7 \%, 6.7 \%$ and $7.1 \%$. The robustness of (4.29) comes from the freedom in the construction of our equilibrium, that is independent of the actual aspect-ratio of the plasma, and redefines the region of interest to satisfy our own aspect-ratio conditions; this improves the accuracy of the successive approximation method and leads to more general properties of the CRECs with one island. Another advantage of this formulation is that we do not need to choose the arbitrary functions or know the particular profiles of the pressure and toroidal magnetic field, improving the generality of the results.

\subsubsection{Safety Factor}

The safety factor will provide us information about the helicity of the magnetic field lines in the equilibrium configuration. In local polar coordinates this can be calculated through

$$
q(\psi)=\frac{1}{2 \pi} \int_{0}^{2 \pi}\left[\frac{r B_{\phi}}{R B_{p}}\right]_{\psi} d \theta
$$

where the integral is to be calculated over a given magnetic surface. Near the magnetic axis, the flux surfaces are almost circular and may be considered as centered circles under integration, also we can consider the toroidal magnetic field as a constant along the small region we are considering. To zero order we can estimate the safety factor by its cylindrical form

$$
q(r)=\frac{r B_{\phi}}{|\nabla \psi|}=\frac{16 a^{2} B_{0}}{\alpha} \frac{\tilde{r}}{|\tilde{\nabla} \Phi|},
$$

with $B_{0}$ the magnetic field at the magnetic axis, $\Phi$ as defined in (4.21) and the values with tilde are dimensionless. This leads simply to

$$
q(r)=\frac{q_{0}}{1+\frac{\eta}{2} r^{2}}
$$

where $q_{0}=2 a^{2} B_{0} / \alpha$. This representation is expected to be valid for $r$ small, away from the critical region, however the predicted divergence at $r_{0}=\sqrt{2 /|\eta|}$ for negative current hollows $(\eta<0)$ actually happens. Recalling that the safety factor may be interpreted as the ratio between the number of toroidal cycles to the poloidal ones for any magnetic line on a given magnetic surface, then the safety factor diverges at the critical surface exactly. This comes from the existence of an hyperbolic point, such that the magnetic lines on the corresponding surfaces will never be able to fulfill a poloidal cycle, like a pendulum in its movement along the separatrix in the phase space. The inversion of the sign after the divergence indicates an inversion in the direction of the poloidal field after the critic 
region (fig. 4.15), that is also expected from the analysis performed in the Section 4.2.2.

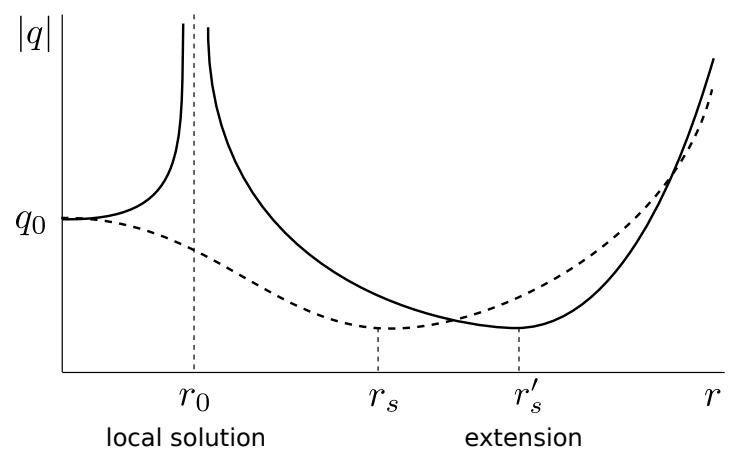

Figure 4.15: Appearance of the safety factor (zero order) about the principal axis for a reversed configuration (continuous curve), and a positive hollow one (dashed curve).

The sign of $q(r)$ in (4.32) keeps the information about the orientation of the poloidal field in a $\phi=$ const. plane. In fig. 4.15 the magnitude of the safety factor in the current reversed case, is compared with the corresponding to a positive hollow current; both are extended to the outer region $(r>O(a))$ by admitting that the current density drops near the plasma edge, this leads to the creation of a stationary point of $q(r)$; the basic characteristic of a shearless surface. It is easy to prove at zero order, that the existence of a current reversal increases the minor radius of the shearless torus, inducing a larger transport barrier for the magnetic lines.

Note that magnetic lines inside the magnetic island does not encircle the principal magnetic axis, so that the safety factor appears to be undefined there; nevertheless, as this is an equilibrium axisymmetric configuration, the magnetic lines on surfaces inside the island have a well defined behavior, encircling the secondary magnetic axis (the one inside the island), for those, the safety factor must be calculated about the corresponding axis. This is of course a first order feature, and that is why it does not appear in the previous calculations.

In the following, an accurate scheme alternative to (4.30) is used to calculate the safety factor numerically. Recall that in this context, the magnetic field may be written as

$$
\boldsymbol{B}=\nabla \psi \times \nabla \phi+\hat{\boldsymbol{\phi}} B_{\phi}
$$

Considering the toroidal field $B_{\phi}$ as a constant in the small region of interest, we can write in the local polar coordinates $(r, \theta), B_{\phi} \hat{\boldsymbol{\phi}}=\nabla\left(B_{\phi} r^{2} / 2\right) \times \nabla \theta$. Defining $\varphi=B_{\phi} r^{2} / 2$, (4.33) becomes

$$
\boldsymbol{B}=\nabla \psi \times \nabla \phi-\nabla \theta \times \nabla \varphi
$$

This puts in evidence the hamiltonian structure of the magnetic field. Comparing with 
(2.17), the Hamilton equations governing the magnetic lines are

$$
\frac{d \varphi}{d \phi}=\frac{\partial \psi}{\partial \theta}, \frac{d \theta}{d \phi}=-\frac{\partial \psi}{\partial \varphi} .
$$

Happily, adaptive symplectic integrators provide accurate numerical solutions to problems of this kind. Using the previously defined constants and dimensionless variables, the problem may be casted like

$$
\frac{d r}{d \phi}=\frac{1}{4 q_{0}} \frac{\partial \Phi}{\partial \theta}, \frac{d \theta}{d \phi}=-\frac{1}{4 q_{0}} \frac{\partial \Phi}{\partial r^{2}}
$$

using (4.21) we are left with the system

$$
\begin{aligned}
\frac{d r}{d \phi} & =-\frac{\epsilon}{4 q_{0}}\left(3+\frac{5}{6} \eta r^{2}\right) r^{2} \sin \theta \\
\frac{d \theta}{d \phi} & =-\frac{1}{q_{0}}\left[\left(1+\eta \frac{r^{2}}{2}\right)+\epsilon\left(\frac{9}{8}+\frac{25}{48} \eta r^{2}\right) r \cos \theta\right] .
\end{aligned}
$$

Note that as $r \rightarrow 0$ we get $d \theta / d \phi \rightarrow-1 / q_{0}$ as expected from the earlier model (the minus sign comes from the choice of the positive sense in the local polar coordinates).

To extract the safety factor profile from the last system, it is integrated numerically for different initial conditions. When the poloidal angle performs one cycle or libration the toroidal angle divided by $2 \pi$ gives the safety factor. In fig. 4.16 the results of this numerical procedure for a particular choice of parameters is depicted. The initial conditions are chosen over the line passing through both elliptic points.
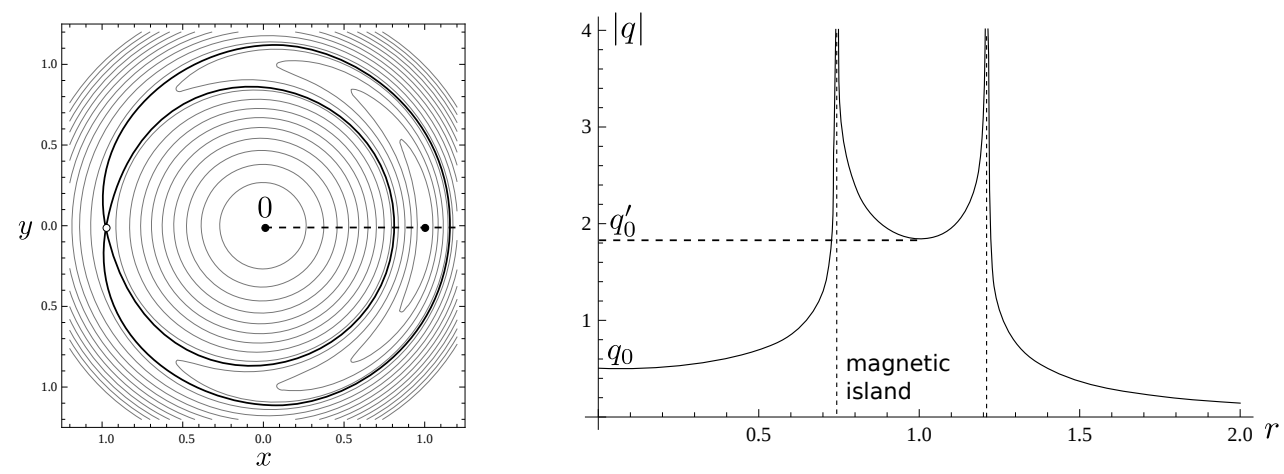

Figure 4.16: Local safety factor for $\eta=-2, \epsilon=0.1$ and $q_{0}=1 / 2$. In the line $\theta=0$.

The minimum in the safety factor inside the island should not be confused with a shearless point, since it corresponds to the value of the local safety factor at the secondary magnetic axis, not to a vanishing shear as one moves transversely to the magnetic surfaces. This method to calculate the safety factor does not provide false divergences at the axisymmetric islands, like in [32], and introduces the possibility of local resonances 
for magnetic surfaces about the secondary axis in the non-axisymmetric case.

\subsubsection{The current clamp hypothesis and the instability conjec- ture}

In the experimental realization of a zero current density region and its subsequent numerical description, it is observed that the current density avoid negative values. Hawkes et.al. in its 2001 PRL [11] claims, "Simultaneous current ramping and application of lower hybrid heating and current drive (LHCD) have produced a region with zero current density (...) However, the core current density is clamped at zero, indicating the existence of a physical mechanism which prevents it from becoming negative.". Although here we deal with a non-equilibrium configuration, the introduced equilibrium is an expected fixed point of the full dynamical system, and the described experimental setup may be away the basins of attraction of our configuration. The mechanism preventing the density to become negative may be the topological breakdown needed for the formation of the magnetic islands; so that, in general, nonested configurations may require a different experimental prelude to be attained. Current reversal configurations are expected to be an extension of the hollow scenarios, a nested topology; however, the formation of a negative current density brings a radical change in the equilibrium geometry.

In the following, our aim is to develop a conjecture, containing a possible mechanism that prevents the formation of a negative current, or makes it difficult to be attained. First of all, recall that our equilibrium configuration exhibit two elliptic points encircled by a self-intersecting circuit $\gamma$ (fig. 4.13). This defines a couple of homoclinic orbits starting and ending at the hyperbolic point. Magnetic lines in critical surfaces are very sensitive to any periodic perturbation of the poloidal flux. In the integrable case, these orbits spend an infinite time (toroidal cycles) near the hyperbolic points without being able to close. From this, in the non-integrable case, these orbits wind at mercy of the periodic perturbations. In fact, periodic perturbations split the homoclinic orbit into an stable and unstable invariant manifolds that cross instead of merge. Of course when a weak perturbation is added, the system is no longer integrable, and only irrational magnetic surfaces survive between layers of chaotic field lines about resonant surfaces.

As the system still deterministic, there is a conservative invertible Poincaré map $T(x)$, that after each iteration gives the subsequent intersections of the magnetics lines with a $\phi=$ const. plane. The stable and unstable manifolds of the hyperbolic point are the collections of every initial condition evolving, to or from, this critical point, respectively. Those manifolds are the same for the Poincaré map. A simple consequence of this is that a single intersection of the stable $\left(W^{s}\right)$ and unstable $\left(W^{u}\right)$ manifolds implies the existence of infinitely many intersections (fig. 4.17 - center). 

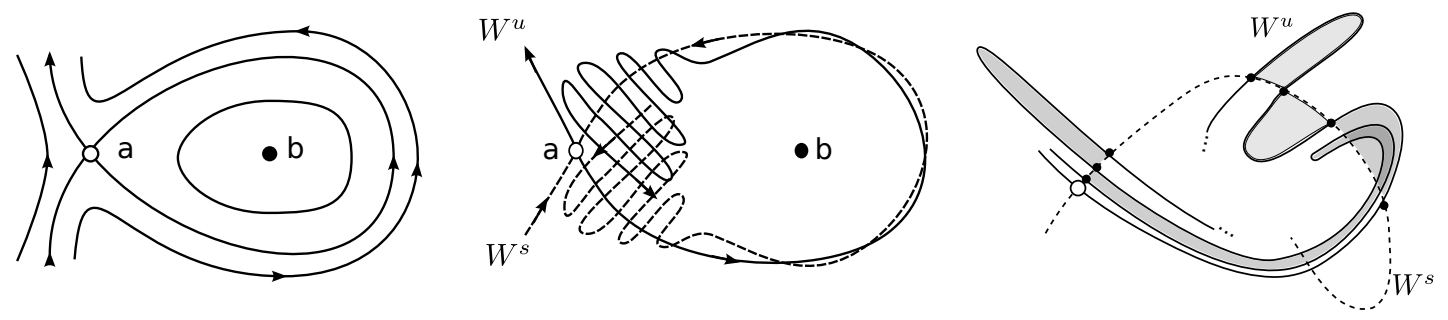

Figure 4.17: Homoclinic scenario in the integrable case (left). Intersections of $W^{s}$ and $W^{u}$ in the non-integrable case (center). Preservation of the lobe area after iteration of the Poincaré map (right).

This property, together with the symplectic area preservation of the hamiltonian systems compound a well understood mechanism for chaos production about the hyperbolic point. In fig. 4.17 (right) we can see how the lobe produced between two intersections of $W^{s}$ and $W^{u}$ is stretched to maintain its area, leading to intersection with an earlier lobe, defining regions containing a densely packaged set of unstable periodic orbits [18].

The homoclinic orbits of the axisymmetric case are easily destroyed by any small perturbation, creating a chaotic sea about the opposite current channels (fig. 4.18). In the rest of the system, rational surfaces (those with $q$ rational) are destroyed to bring resonant islands of different sizes (usually small for $q \neq 1$ ), and most of the irrational ones are slightly deformed but preserve their topology.
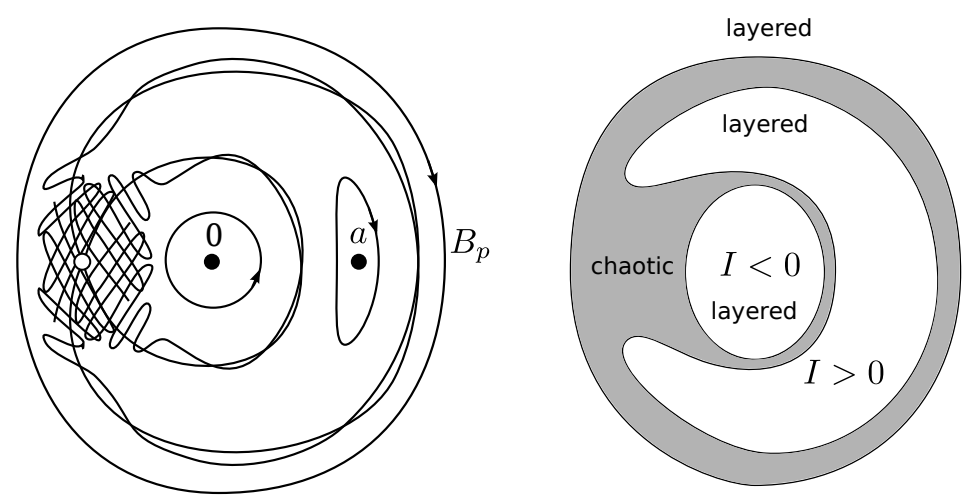

Figure 4.18: Homoclinic chaos about the hyperbolic point and zones of total and partial destruction of magnetic surfaces.

Periodic perturbations are intrinsic to the tokamak design. For instance, the discreteness of the toroidal field coils makes the plasma a little bumpy, and the existence of diagnostic elements at fixed points in the torus introduces small variations in the physical quantities that are periodic in the hamiltonian formulation of the field, etc. It is then important to consider this unavoidable effects specially when hyperbolic points appears in the equilibrium, since they will be an important source of chaotic behavior of the magnetic lines. 
To illustrate this point, we can integrate numerically the equations for the magnetic lines under a small disturbance of the magnetic poloidal flux. In this case the perturbation behaves like $\delta \Phi \propto r^{2} \sin \phi$, the details of its derivation can be found on the Appendix of this work. This represents a single toroidal mode of a fluctuation in the poloidal flux, corresponding to a local bump in the magnetic surfaces due the lack of some toroidal field coils in a small region of the tokamak. This introduces a small correction in the hamilton equation for the poloidal angle of the magnetic line

$$
\frac{d \theta}{d \phi}=\frac{1}{4 q_{0}} \frac{\partial}{\partial r^{2}}\left(\Phi_{0}+\delta \Phi\right)=\frac{1}{4 q_{0}}\left(\frac{\partial \Phi_{0}}{\partial r^{2}}+\varepsilon k \cos \phi\right)
$$

making the system non-autonomous, and non-integrable. The addition of such perturbation assumes the equilibrium still existing, and most of its basic properties does not change drastically (like the pressure and current profiles). However dissipative events, like reconnection of magnetic lines, may alter the equilibrium topology in a time-dependent description.

After the introduction of this correction, the equations ruling the evolution of the magnetic field lines are

$$
\begin{aligned}
& \frac{d r}{d \phi}=-\frac{\epsilon}{4 q_{0}}\left(3+\frac{5}{6} \eta r^{2}\right) r^{2} \sin \theta \\
& \frac{d \theta}{d \phi}=-\frac{1}{q_{0}}\left[\left(1+\eta \frac{r^{2}}{2}\right)+\epsilon\left(\frac{9}{8}+\frac{25}{48} \eta r^{2}\right) r \cos \theta+\varepsilon \cos \phi\right]
\end{aligned}
$$

This simple non-integrable system exhibits the main features of homoclinic chaos exposed previously, and the control parameter $\varepsilon$ defines the size of the chaotic region between the current channels.

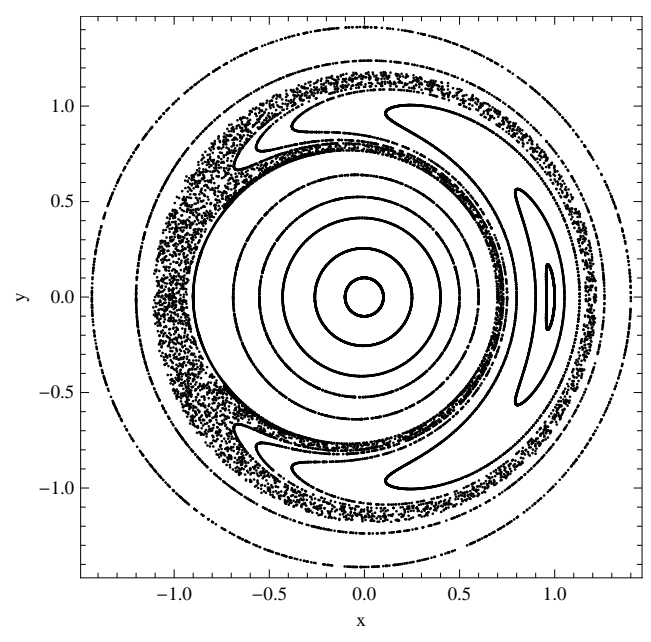

Figure 4.19: Poincaré section of the magnetic lines for a simple perturbation with intensity $10 \%$ to the equilibrium with $\eta=-2, \epsilon=0.1$. 
In fig. 4.19 we plot a numerical Poincaré section of this system, where we can clearly appreciate the coexistence of quasi-periodic and chaotic regions. In regard to homoclinic chaos, it is not really important the particular form of the perturbing flux, the horseshoe mechanism [18] is not very sensitive to the variation of the perturbation in the radial direction (i.e. the action variable). Now that we are conscious of the intimate relation between hyperbolic points and chaos, lets address a quite simple, but remarkable property of chaotic magnetic fields in perfectly conducting, time independent plasmas.

In equilibrium (it does not matter if integrable or not) the equation $\nabla p=\boldsymbol{j} \times \boldsymbol{B}$ is satisfied. This imply $\nabla p \cdot \boldsymbol{B}=0$, in words, the pressure is constant along a magnetic line. If such line is chaotic, it pierces densely a given region of the Poincaré section (fig. 4.20 - left), bringing at each point the same value of the pressure. From this, $\nabla p=0$ and the kinetic forces vanishes in the chaotic regions between the invariant surfaces. Obviously, the equilibrium theory does not provide means to calculate the pressure in the chaotic region; but assuming that it is constant through it, we have some interesting consequences.

First, we can not guarantee that the pressure at the last invariant surfaces in the edge of the current channels is the same as that in the chaotic region; in fact, as we vary the perturbation intensity, homoclinic chaos proceeds from the separatrix absorbing invariant surfaces in both sides. Those invariants are not consumed in an ordered fashion relative to the pressure, but to the safety factor.

Now, the small perturbation to the poloidal magnetic flux is a smooth function, then, it is related to a smooth magnetic field. The total field $\boldsymbol{B}+\delta \boldsymbol{B}$ is smooth as well, even if the magnetic lines are chaotic. This means that there are no discontinuities in the magnetic field, at least inside the plasma.

From the last two paragraphs, in the non-axisymmetric case we may have discontinuities in the kinetic pressure with no discontinuity in the magnetic field. However, in equilibrium, the boundary condition $\delta\left(p+B^{2} / 2 \mu_{0}\right)=0$ must be verified at any magnetic surface, including the one separating the chaotic from the regular regions. This suggests that the described situation does not correspond to an equilibrium one, since there are unbalanced forces acting on the boundaries of the current channels.

To recover the equilibrium under this circumstances a somewhat subtle element must be introduced. A surface current at the last invariant surface in the form $\mu_{0} \boldsymbol{K}=\hat{n} \times \Delta \boldsymbol{B}$; where $\Delta \boldsymbol{B}$ is a discontinuity in the magnetic field that balances the step in the kinetic pressure. However, $\boldsymbol{\Delta} \boldsymbol{B}$ is not provided by the model and should be obtained from a different approach. Another possible consideration, is that in the non-axisymmetric case, the pressure plateau matches exactly the pressure in the last invariant surfaces of the current channels; but this introduces a strong restriction on the types of axisymmetric equilibria that remains stable under azimuthal disturbances. Clearly, it appears more 
reasonable to restrict the types of integrable CRECs that assume self-sustained surface currents in the non-axisymmetric case.

\subsection{Concluding Remarks}

Due to the pressure flattening the equilibrium in the central region must be attained only from magnetic forces. This is a very unfavorable situation, since we don't have full domain of the internal magnetic field. In fact, the contribution to the magnetic field from the positive current channel tends to eject the negative one (fig. 4.20 - right) and vice versa. We can think of a downwards external field to balance the situation, but variations on the vertical field may alter drastically the overall equilibrium outside the region of interest, subsequently changing the conditions under which the internal equilibrium was reached. From the lack of kinetic force in the chaotic region, and forces at the boundary of the current channels, we may think that the system is left in a self-organizing situation, where the invariant surfaces are able to move seeking for a more favorable configuration.
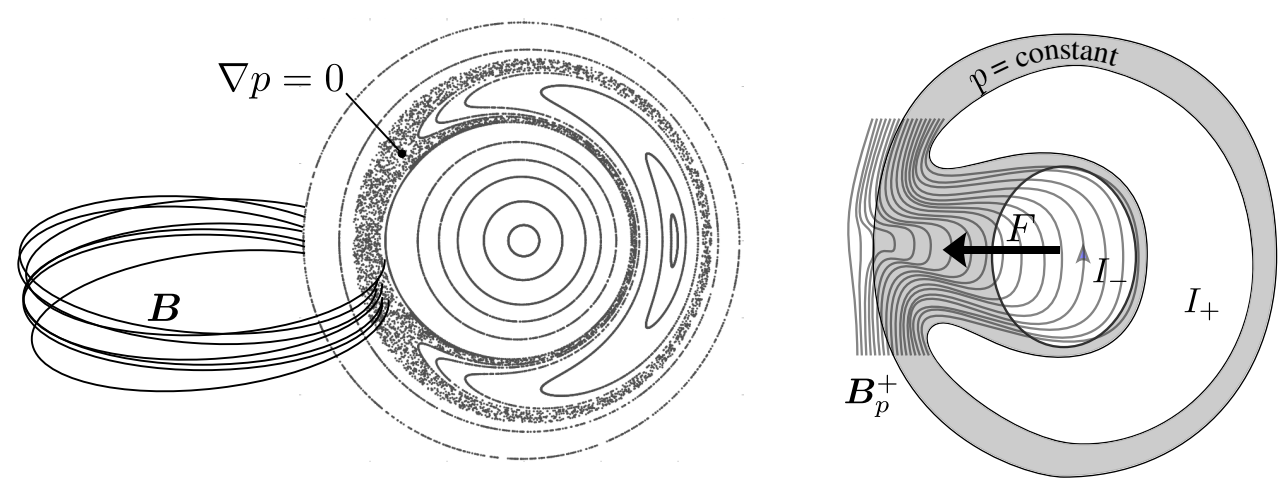

Figure 4.20: A chaotic field line fills a region with constant pressure, where the kinetic forces can't balance the magnetic ones produced by one current channel on the other.

Also, it is worth to mention that in developing the MHD equilibrium equations, small terms related to non-uniformities of the velocity field, and tensorial structure of the pressure were neglected. This additional terms may enable the existence of equilibrium configurations where the kinetic pressure is not uniform along the magnetic lines, conceiving $\nabla p \neq 0$ in chaotic regions; so that the force balance may be reached from this small terms [35]. Also, in a more general picture, reconnection of magnetic lines may alter the global topology by altering the magnetic surfaces about the hyperbolic point as it transforms the magnetic energy into kinetic one [36], leading to an unstable situation. 


\section{Chapter 5}

\section{Conclusions}

The response of the equilibrium topology to the existence of a small reversed toroidal currents was studied. From a simple geometrical approach, based on the Maxwell equations and integrability of the magnetic field lines, we showed the incompatibility with usual nested configuration. In summary, a nested topology requires strong symmetries of the equilibrium, restricting severely the generality of the results. In a constructive fashion, we showed that the CRECs lead naturally to nonested magnetic surfaces, or better, to several families of nested magnetic surfaces separated by intersecting or self-intersecting critical surfaces. After this, we obtained the one-island topology from the Grad-Shafranov equation. Here, a prototypical parabolic current with a small negative minimum removes the choice of arbitrary functions. To solve the G-S equation we have introduced a modified successive approximations mechanism, wherein the aspect ratio was defined from the "region of interest" instead of the plasma radius, making the solution a local one.

From the local solution, we found the characteristic size of the island as a simple function of the critical radius, where the island is formed. We also tested the result with some published one-island CRECs. These where obtained from different numerical treatments or a linearizion of the G-S equation. Reasonable accuracy (about 7\%) prevailed after changes of the somewhat arbitrary size of the region of interest. As was pointed before, the equilibrium structure have some strong topological bounds, and the particular choice of a given pair of source functions does not affect deeply the main features of the local equilibrium; this is why our simplified model gives good account of other results.

Finally, the necessary existence of hyperbolic points in the relevant domain of the CRECs, is pointed to be an important source of chaotic fields in the nonintegrable case. The chaotic magnetic lines, mainly located about the hyperbolic point, creates (in first approximation) a flat pressure region between the opposite current channels, demanding a vanishing of the product $\boldsymbol{j} \times \boldsymbol{B}$ in the chaotic region. From this, the equilibrium between the channels must be attained just from the internal magnetic fields, an overwhelming 
technological challenge if the system does not evolve naturally to such state. Moreover, the current channels naturally tends to throw out each other and without the kinetic force balancing the equation the scenario is probably unstable. This conjectures are in agreement with most of experimental observations, that suggest that the toroidal current is clamped at zero, and some "unknown" mechanism prevents it from becoming negative. 


\section{Chapter 6}

\section{APPENDIX}

\subsection{Poloidal flux perturbation model}

In tokamaks, a discrete set of current loops around the toroidal chamber produces a toroidal magnetic field inside the plasma. This discrete structure make the system nonaxisymmetric, introducing small perturbations to the toroidal magnetic field. However, the effect due to discreteness of toroidal field coils is small compared to the introduced by the absence of some coils; this absence is most times necessary for positioning diagnostic components.

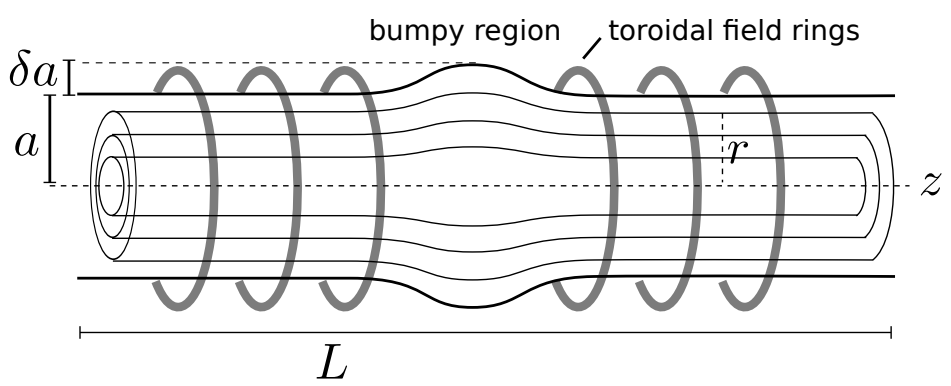

Figure 6.1: Bumpy region of the magnetic surfaces due to toroidal field weakening

In the case of an infinite cylindrical plasma, the absence of some coil creates a small bumpy region where the plasma edge grows (fig. 6.1). However, the system remains axisymmetric about the $z$-axis, so that the problem remains integrable and bumpy versions of the magnetic surfaces continue to exist. To simplify this model, lets assume that the current density in $z$ is uniform in the plane $z=$ const. Then to conserve the plasma current we require $j_{z}$ to be a function of $z$ only. From the Ampere's law is easy to get

$$
B_{\theta}(r, z)=\frac{\mu_{0}}{2} j_{z}(z) r
$$


In a toroidal geometry the poloidal field can be written as

$$
\boldsymbol{B}_{p}=\nabla \psi \times \nabla \phi
$$

that in the large aspect ratio approximation gives

$$
B_{\theta}=\frac{1}{R} \frac{d \psi}{d r}
$$

Comparing with (6.1) and integrating in $\mathrm{r}$ we have

$$
\psi=\frac{\mu_{0} R}{4} j_{z}(z) r^{2}
$$

where the poloidal flux was considered zero at the magnetic axis, and $R$ was kept constant during the integration. Finally, we can write the current density like:

$$
j_{z}(z)=\frac{I_{p}}{\pi(a+\delta a(z))^{2}} \approx \frac{I_{p}}{\pi a^{2}}\left(1-2 \frac{\delta a(z)}{a}\right)
$$

where $\delta a(z)$ is a small correction to the plasma edge radius, as a function of $z$. Defining $\delta a\left(z_{0}\right)=\delta a$ as the maximum deformation of the edge, and defining $\varepsilon=\delta a / a$, inserting (6.5) into (6.4) we get

$$
\psi(r, z)=\frac{\mu_{0} I_{p} R}{4 \pi}\left(1-\varepsilon f\left(z, z_{0}\right)\right) \tilde{r}^{2},
$$

where $f\left(z, z_{0}\right)$ is 1 at $z_{0}$ or in the plasma belly, and zero away from it; and $\tilde{r}=r / a$ is the nondimensional radius. From this expression is easy to recognize the correction $\delta \psi$ to the poloidal flux due to the belly in the plasma. Returning to the toroidal geometry we write

$$
\delta \psi(r, \phi)=\varepsilon \psi_{a} \tilde{r}^{2} f(\phi)
$$

This last equation is written in canonical units of flux $\psi_{a}=\mu_{0} I_{p} R / 4 \pi$, and $f(\phi)$ is now a function of the toroidal angle. The modulation function $f(\phi)$ may be expanded in the Fourier basis, in which its most important component is the $n=1$; so that a reasonable choice for the perturbation to the poloidal flux due to a discontinuity of the external loops is

$$
\delta \psi(r, \phi)=\varepsilon k \tilde{r}^{2} \sin (\phi)
$$

where $k$ contains the Fourier amplitude in canonical flux units. 


\section{References}

[1] B. D. Bondarenko. Role played by O. A. Lavrent'ev in the formulation of the problem and the initiation of research into controlled nuclear fusion in the USSR. Physics Uspekhi, 44(8):844, 2001.

[2] J. P. Freidberg. Ideal magnetohydrodinamic theory of magnetic fusion systems. Reviews of Modern Physics, 54(3):801, 1982.

[3] L.E. Zakharov. Low recycling regime in ITER and the LiWall concept for its divertor. Journal of Nuclear Materials, 363-365:453, 2007.

[4] E. Joffrin et al. Advanced tokamak scenario, developments for the next step. Technical Report PR(07)36, EFDA-JET.

[5] A. A. Galeev. Journal of Experimental and Theoretical Physics, 59:1378, 1970.

[6] F.M. Levinton et al. Physical Review Letters, 75:4417, 1995.

[7] S.J. Strait et al. Physical Review Letters, 75:4421, 1995.

[8] T. Fujita. Physical Review Letters, 78:2377, 1996.

[9] T. Kroetz. Linhas de Campo Magnético Caóticas em Tokamaks. Tese de Mestrado Instituto Tecnológico de Aeronáutica, São Jose dos Campos, 2006.

[10] J da Fonseca. Barreiras de Transporte em Plasmas e Mapas Simpléticos Não-Twist. Tese de Mestrado - Universidade de São Paulo, São Paulo, 2011.

[11] N. C. Hawkes et al. Observation of zero current density in the core of jet discharges with lower hybrid heating and current drive. Physical Review Letters, 87(11):115001, 2001.

[12] P. Rodrigues and J. Bizarro. Topology of plasma equilibrium with toroidal current reversal. Physics of Plasmas, 19:012504, 2012.

[13] F. F. Chen. Plasma Physics and Controlled Fusion - 2nd Ed. Springer, 2006. 
[14] J. Wesson. Tokamaks. Clarendon Press, Oxford, 1987.

[15] V. D. Shafranov. Plasma equilibrium in a magnetic field. Reviews of Plasma Physics, 2:103, 1966.

[16] R. B. White. Toroidally Confined Plasmas. Imperial College Press, 2006.

[17] R. Fitzpatrick. Plasma physics. http://farside.ph.utexas.edu/teaching/plasma, 2011 .

[18] J. Guckenheimer and P. Holmes. Nonlinear Oscillations, Dynamical Systems, and Bifurcations of Vector Fields. Springer, 1983.

[19] J. D. Jackson. Classical Electrodynamics 3rd Ed. John Wiley \& Sons, 1998.

[20] V. I. Arnold. Mathematical Methods of Classical Mechanics - 2nd Ed. SpringerVerlag, 1989.

[21] H. Goldstein, C. Poole, and J. Safko. Classical Mechanics, 3rd Ed. Addison Wesley, 2000 .

[22] P. Cvitanović, R. Artuso, R. Mainieri, G. Tanner, and G. Vattay. Chaos: Classical and Quantum. ChaosBook.org, Niels Bohr Institute, Copenhagen, 2009.

[23] J. Litchenberg and M. A. Lieberman. Regular and Chaotic Dynmics. Springer-Verlag, 1992.

[24] J. José and E. Saletan. Classical Dynamics, a contemporary approach. Cambridge, 1998.

[25] P.J. McCarthy. Analytical solutions to the Grad-Shafranov equation for tokamak equilibrium with dissimilar source functions. Physics of Plasmas, 6(9):3554, 1999.

[26] S.B. Zheng. Analytical tokamak equilibrium for shaped plasmas. Physics of Plasmas, 3(3):1176, 1996.

[27] G. Arfken. Mathematical Methods for Physicists, 2nd ed. Academic Press, 1985.

[28] R.M.O. Galvão. Teoria Magnetoidrodinâmica, Equilíbrio e Estabilidade. Notas de aula, ITA-CTA, 1989.

[29] F.A. Marcus, T. Kroetz, M. Roberto, I.L. Caldas, E.C. da Silva, R.L. Viana, and Z.O. Guimarães-Filho. Chaotic transport in reversed shear tokamaks. Nuclear Fusion, 48:024018, 2008. 
[30] J. Portela, I.L.Caldas, R. L. Vianna, and P.J. Morrison. Diffusive transport through a nontwist barrier in tokamaks. International Journal of Bifurcation and Chaos, $17(5): 1589,2007$.

[31] S. Wang. Theory of tokamak equilibria with central current density reversal. Physical Review Letters, 93(15):155007, 2004.

[32] A.A. Martynov and S. Medvedev. Tokamak equilibria with reversed current density. Physical Review Letters, 91(8):085004, 2003.

[33] C. Martins, M. Roberto, I. L. Caldas, and F. L. Braga. Analytical solutions for tokamak equilibria with reversed toroidal current. Physics of Plasmas, 18:082508, 2011.

[34] S. Medvedev et al. Tokamak equilibria with reversed current density. Proceedings of the 20th EPS Conference on Controlled Fusion and Plasma Physics, Lisbon, 17C(part IV):1729, 1993.

[35] J.A. Krommes and A. Reiman. Plasma equilibrium in a magnetic field with stochastic regions. PPPL-Report, 4401, 2009.

[36] D. Biskamp. Magnetic reconnection via current sheets. Physics of Fluids, 29:1520, 1986. 